\title{
Speaker presentations
}

\section{S-01 The importance of breast cancer research from a patient's view: the voices and visions of advocates}

\section{S Leigh}

Past President, National Coalition for Cancer Survivorship, Cancer Survivorship Consultant, Tucson, Arizona, USA

While advances in science and technology have increased options for treating breast cancer, current social trends have changed the way people deal with this disease. Women in the United States are no longer simply passive patients, but rather they are survivors, advocates and activists who are speaking up for themselves and speaking out for issues relevant to the treatment and prevention of breast cancer.

As the discoveries of basic science have been translated to better clinical treatment, a new sense of hope has emerged. Quality of life now shares the spotlight with quantity of life as breast cancer has shifted from an acute to a chronic condition and as the numbers of long-term survivors increase. While this new population tends to have more optimistic expectations for survival, they are also expressing concerns about issues affecting their lives through and beyond treatment. These issues include, but are not limited to, such concerns as efficient and accurate diagnosis, the complexity of treatment decisions, access to quality cancer care, informed consent, privacy issues, availability of supportive care treatments, and effective communication skills, especially with their physicians. Survivors are also concerned about the impact of their disease on spouses and family, on fertility and sexuality issues, on their employment and (in the USA) insurability, and on their long-term survival. The identification of these increasing issues has given rise to a consumer movement that encourages a shift away from powerless victim to empowered survivor.

Historically, breast cancer advocates asked for increased educational and supportive care resources. As the survivorship movement matured, new responsibilities and differing agendas arose amongst these groups. Some organizations defined their mission as one that would raise funds to support scientific research. Others felt compelled to raise awareness about early detection and treatment, controversial environmental issues, and prevention or risk reduction. A few organizations later entered the more political arenas and began lobbying for issues related to health care delivery, clinical trials access, and quality cancer care. Meanwhile, these many and varied missions are all helping to define an international agenda for breast cancer research and care, to guarantee the inclusion of consumer voices in most levels of decision-making, and to create partnerships between patients with breast cancer and the professionals who care for them.

\section{S-02 Abstract not submitted for publication}

\section{S-03 Abstract not submitted for publication}

S-04 Genetic testing for BRCA1 and BRCA2 mutations - ready for implementation?

\section{BL Weber}

University of Pennsylvania, Philadelphia, PA, USA

With the discovery of $B R C A 1$ and BRCA2, testing for germline mutations became a possibility. However, there are several questions that must be considered if genetic testing is to be widely implemented. First, who should have the test - are there defined groups at increased risk? Second, is the laboratory technically capable of accurate testing and with what sensitivity and specificity? Are the test results interpretable? Finally, is there clinical utility to the test? That is, are there interventions as a result of the test that will benefit the patient, and do the benefits outweigh the risks? 
At least partial answers to these questions are now available. There are well-established methods of identifying mutations, and there are known founder mutations that simplify testing in some populations. In particular, there are data that suggest that screening all Ashkenazi Jewish women for the three founder mutations in this group may significantly reduce deaths from ovarian cancer in this population. Direct sequencing and heteroduplex analysis are both methods with sensitivity well over $90 \%$ for coding region and splice site mutations; however, the problem of genomic rearrangements in $B R C A 1$ remains. Variants of uncertain significance remain a problem, particularly in $B R C A 2$, but truncating mutations are clearly associated with a markedly increased risk of breast and ovarian cancer. Perhaps most importantly, recent work is beginning to provide justification for prevention strategies for both breast and ovarian cancer, as well as evidence that genetic testing is well-tolerated psychologically. Finally, most Western countries have addressed the issue of genetic discrimination and offer protection through either nationalized health services or federal legislation. In summary, the past five years have yielded advances in all areas pertaining to genetic susceptibility testing, and the promise of cancer prevention associated with the isolation of $B R C A 1$ and $B R C A 2$ is becoming a reality.

\section{The pathology of inherited breast tumours}

\section{Stratton}

Institute of Cancer Research, UK

There is now a considerable body of information pertaining to the histopathological appearances of breast cancers arising in multiple case families due to germline mutations in breast cancer susceptibility genes. The evidence indicates that cancers in BRCA1 and BRCA2 mutation carriers differ overall in morphological indices seen by $\mathrm{H}+\mathrm{E}$ staining from each other, and also from age-matched cases unselected for family history. BRCA1 cancers differ much more substantially from controls than BRCA2 cancers and overall are of higher grade. Differences between these groups are also seen immunohistochemically for a number of proteins. Notably, BRCA1 cancers are rarely ER positive compared to BRCA2 and controls. Cancers from families not due to either known gene but which are likely to be due to other, currently unknown susceptibility genes, also differ from BRCA1, BRCA2 and age-matched control cancers. These cancers are generally low grade lesions with the suggestion of an excess of lobular carcinoma cases. The significance of these histological differences with respect to prognosis remains controversial.

\section{Molecular characteristics of inherited breast tumors}

\section{Å Borg, IA Hedenfalk, J Vallon-Christersson, N Loman, O Johannsson, H Olsson, DJ Duggan, Y Chen, M Bittner, O-P Kallioniemi and JM Trent \\ Department of Oncology, Lund University, SE-221 85 Lund; Sweden and Cancer Genetics Branch, National Human Genome Research Institute, NIH, Bethesda, MD 20892, USA}

Germline mutations in genes involved in DNA doublestrand break repair (DSBR) and DNA damage-induced checkpoint activation are associated with chromosomal breakage syndromes and (breast) cancer predisposition. These genes include TP53, CHK2, ATM, NBS1, Mre11 and the two major breast-cancer susceptibility genes $B R C A 1$ and BRCA2. Breast tumors from BRCA1 and $B R C A 2$ mutation carriers have explicit histopathological features and genetic alterations, distinct from other forms of inherited (BRCAx) and sporadic breast cancer. This suggests that transformation of DSBR-deficient cells follows abrogation of specific cell-cycle control and apoptosis mechanisms, and results in genetic instability and tumor progression along distinguishable pathways. Comparative genomic hybridization (CGH) analysis may give hints to the location of such genes by showing frequent loss of chromosome $4,5 q, 12 q, 13 q$ and $X q$ in BRCA1 tumors, and of $1 p, 3 p, 6 q, 8 p, 9 p, 11 q, 13 q$ and $X q$ in $B R C A 2$ tumors. Frequent copy number gains are seen at
$1 q, 6 p, 8 q, 10 p, 16 p$ and $17 q$ in $B R C A 1$ tumors, and at $1 \mathrm{q}, 8 \mathrm{q}, 16 \mathrm{p}, 17 \mathrm{q}, 19$ and $20 \mathrm{q}$ in BRCA2 tumors. By extending the analyses to the level of gene expression, using cDNA microarrays containing 6500 sequence-verified human genes or ESTs, we have shown that BRCA1 and BRCA2 tumors can be separated into distinct clusters by multi-dimensional scaling and hierarchical dendrogram analysis of expression data. Genes consistently upor downregulated in each group of inherited breast cancer have been identified, and will be evaluated as diagnostic tools in new sets of tumors, also on the level of protein expression. The presumably heterogeneous group of $B R C A x$ breast tumors exhibits, in general, a less aggressive phenotype, being typically of low malignant grade and steroid receptor-positive status. Further characterisation of gene alteration and expression profiles in these tumors may be used as a complement to traditional linkage analysis in the search for additional breast cancer susceptibility genes. 
S-07 Other cancers in BRCA1 and BRCA2 mutation carriers: implications for counselling and follow up

B Ponder

CRC Department of Oncology, University of Cambridge, Cambridge, UK

Data come from the Breast Cancer Linkage Consortium. The BRCA1 estimates (from 1993) are being updated. The overall risk of ovarian cancer was estimated as $30 \%$ by age 60 (but the data suggested the possibility, subsequently supported by mutation data, of heterogeneity, with two groups of families with higher and lower risks of ovarian cancer), and 3- and 4-fold increases in risk of prostate and colorectal cancer respectively, corresponding to absolute risks of about $5-10 \%$ by age 70 . The BRCA2 estimates are more recent and so based on more extensive data. The estimated cumulative risk of ovarian cancer is $0.4 \%$ by age 50 and $27 \%$ by age 70 (again with evidence of heterogeneity from mutation studies); statistically significant elevated risks are also observed for prostate cancer (overall RR 4.65 [7.33 below age 65]; absolute risk $7.5 \%$ by age 70 ); pancreatic cancer (RR 3.51 [5.54 below age 65 ]; absolute risk $2 \%$ by age 70 ), gall bladder and biliary cancer (RR 4.97), stomach cancer (RR 2.59), malignant melanoma (RR 2.58) and cancer of the oropharynx (RR 2.26, 95\% Cl 1.09-4.58). There was no significant increase in risk of colorectal cancer. The estimated cumulative risk of male breast cancer is $2 \%$ by age 70 , but with very wide confidence limits.

These overall risks will differ in individual cases according to the specific BRCA mutation, and genetic and nongenetic modifiers. Except possibly for the protective effects of OC use on ovarian cancer, this information is not ready to be translated into clinical practice. The main controversy is around screening for colorectal and prostate cancer. The balance of risks and benefits is not known for either; there is no consensus; a BCLC study of prostate screening is proposed and a colorectal study in $B R C A 1$ carriers may be appropriate if the risks are confirmed.

S-08 The role of coactivators in oestrogen action

\section{Brown and JF de Mora}

Department of Adult Oncology, Dana Farber Cancer Institute and Harvard Medical School, Boston, MA 02115, USA

Several classes of coregulatory molecules are felt to play important roles in cell-type specific responses to oestrogens. These ER coactivators include members of the SWI2/SNF2 chromatin remodelling complexes, histone acetyltransferases such as p300/CBP, and p160 factors of the SRC-1 family. We sought to understand more fully how growth factors modulate oestrogen receptor activity in both normal oestrogen physiology and the pathogenesis of breast cancer. Growth factors are known to stimulate the ligand-independent activity of ER through the activation of MAPK and the direct phosphorylation of ER. We have now found that the transcriptional stimulatory activity of the p160 factor AIB1, a gene amplified preferentially in ER-positive breast cancers, is enhanced by MAPK. We show that AIB1 is a phosphoprotein in vivo and can be phosphorylated in vitro by MAPK. Finally we observe that MAPK activation of AlB1 stimulates the recruitment of $\mathrm{p} 300$ and associated histone acetyltransferase activity. These results suggest that the ability of growth factors to modulate oestrogen action may be mediated through MAPK activation of the nuclear receptor coactivator AIB1. In addition they suggest a potential point of cross-talk between growth-factor signalling pathways and oestrogen signalling in ER-positive breast cancers.

\section{S-09 Growth regulation and steroid hormone resistance in breast cancer}

KB Horwitz

University of Colorado School of Medicine, Endocrinology Division, Denver, CO 80262, USA

Our research focuses on breast cancer, and how the steroid hormone agonists - estradiol and progesterone enhance growth of these tumors. Therefore, their treatment often involves the use of steroid antagonists, which interfere with deleterious effects of the agonists. Although tumors often respond well to antagonists initially, and undergo remission, eventually tumors acquire resistance to antagonists and resume growing. I will discuss studies dealing with growth regulatory mechanisms of progesterone, focusing on the role of cyclins; cyclin-dependent kinases and cdk inhibitors; and cross-talk between progesterone and epidermal growth factor (EGF) signaling.
The latter involves analysis of mechanisms by which progesterone and EGF cooperate to activate mitogen-activated protein kinase (MAPK) and STAT signaling pathways, and regulate transcription of the cdk inhibitor, p21. Additionally we show that MAPK phosphorylation of progesterone receptors, at serine 294, leads to liganddependent receptor downregulation by the ubiquitin-26S proteasome pathway. I will also describe the isolation and characterization of transcriptional coactivators and corepressors that either enhance or inhibit transcription by antagonist-occupied steroid receptors. We test the idea that the ratio of these coregulators determines whether 
tamoxifen is inhibitory or not, using breast cancers taken from tamoxifen-responsive and -resistant patients.

\section{References}

Jackson TA et al: Mol Endocrinol 1997, 11:693-705.

Groshong S et al: Mol Endocrinol 1997, 11:1593-1607.

Lange CA et al: J Biol Chem 1998, 273:31308-31316.

Richer JK et al: J Biol Chem 1998, 273:31317-31326.

Lange CA et al: Proc Natl Acad Sci 2000, in press.

\section{S-10 Abstract not submitted for publication}

\section{S-11 Estrogen receptors $\alpha$ and $\beta$ in the rodent mammary gland}

S Saji*, EV Jensen*, S Nilsson†, T Rylander*, M Warner* and J-Å Gustafsson*

${ }^{*}$ Department of Medical Nutrition and Biosciences, Karolinska Institute, NOVUM Huddinge University Hospital, S-14186

Huddinge, and 'KaroBio AB, NOVUM, S-141 86 Huddinge, Sweden

An obligatory role for estrogen in growth, development, and functions of the mammary gland is well established, but the roles of the two estrogen receptors remain unclear. With the use of specific antibodies, it was found that both estrogen receptors, ER $\alpha$ and $E R \beta$, are expressed in the rat mammary gland, but the presence and cellular distribution of the two receptors are distinct. In prepubertal rats, ER $\alpha$ was detected in $40 \%$ of the epithelial cell nuclei. This decreased to $30 \%$ at puberty and continued to decrease throughout pregnancy to a low of $5 \%$ at day 14 . During lactation there was a large induction of ER $\alpha$ with up to $70 \%$ of the nuclei positive at day 21. Approximately $60-70 \%$ of epithelial cells expressed
ER $\beta$ at all stages of breast development. Cells coexpressing $\mathrm{ER} \alpha$ and $\mathrm{ER} \beta$ were rare during pregnancy, a proliferative phase, but they represented up to $60 \%$ of the epithelial cells during lactation, a postproliferative phase. Western blot analysis and sucrose gradient centrifugation confirmed this pattern of expression. During pregnancy, the proliferating cell nuclear antigen was not expressed in ER $\alpha$-positive cells but was observed in 3-7\% of ER $\beta$-containing cells. Because more than $90 \%$ of ERß-bearing cells do not proliferate, and $55-70 \%$ of the dividing cells have neither $E R \alpha$ nor $E R \beta$, it is clear that the presence of these receptors in epithelial cells is not a prerequisite for estrogen-mediated proliferation.

\section{S-12 Clinical translation of progress in molecular endocrinology}

\section{Dowsett}

Academic Department of Biochemistry, The Royal Marsden Hospital, Fulham Road, London SW3 6JJ, UK

There has been substantial recent progress in our understanding of the molecular mechanism of oestrogen action, most particularly by the discovery of (i) a second ER (ii) the role of co-repressors/co-activators (iii) the importance of conformational change of ER. This has provided insight into the mode of action of hormonal drugs for breast cancer, and prompted new ideas about potential resistance mechanisms, new strategies for treatment and prevention, and the development of new drugs. Differential conformational change of ER by SERMs seems to determine the specific binding of the receptor to particular co-activators/co-repressors of gene transcription. Perturbation of this molecular system can provide cells resistant to tamoxifen through an increased agonist response. There are clinical data to support an increased agonist response of tamoxifen as a resistance mechanism in breast cancer, but there are few clinical laboratory data to support aberrant co-activator/co-repressor expression as an important mechanism. Recent in vitro studies indicate that MCF7 cells may become resistant to oestrogen deprivation by acquired hypersensitivity to oestrogen. There are clinical data to support this mechanism, and new clinical trials have been designed to determine whether this phenomenon can be utilised in sequential therapy. To achieve optimal clinical exploitation of the progress in molecular endocrinology, there is a need for novel clinical trial design, which will utilise imaging and molecular pathological techniques for assessing the molecular response of tissues. Neoadjuvant treatment of breast cancer offers unique advantages for such studies. Treatment-induced changes in proliferation are a useful intermediate endpoint for the evaluation of molecular relationships in breast carcinomas in vivo and for the assessment of drugs effecting these relationships.

S-13 The EGF receptor family as targets for breast cancer therapy

\section{J Baselga \\ Hospital General Universitari Vall d'Hebron, Barcelona, Spain}

Breast tumors express high levels of type I receptor tyrosine kinases and their ligands. This receptor family is composed of four homologue receptors; the epidermal growth factor receptor (ErbB1/EGF receptor/HER1), ErbB2 (HER2/neu), ErbB3 (HER3), and ErbB4 (HER4). These receptors are composed of an extracellular binding domain, 
a transmembrane lipophilic segment, and an intracellular protein tyrosine kinase domain with a regulatory carboxyl terminal segment. Several lines of evidence suggest that these receptors are optimal targets for new anti-cancer agents, and a series of monoclonal antibodies are currently being evaluated both in the laboratory and in the clinic. Agents currently under study include monoclonal antibody (MAb) C225 directed at the EGFR, tratstuzumab (Herceptin $^{\circledR}$ ) directed at the HER2 receptor, and a new family of specific EGFR tyrosine kinase inhibitors.

Anti-EGFR MAb 225 prevents the binding of the ligands to the EGFR, blocks ligand-induced activation of the receptor, and inhibits the growth of cancer cells both in tissue culture and in human tumor xenografts. Anti-EGFR MAb 225 greatly enhances the antitumor effects of chemotherapeutic agents active against breast cancer, such as taxol and dox- orubicin. A human:murine 'chimeric' antibody (C225) has been produced with comparable affinity and antitumor activity that allows the administration of repeated doses of MAb either alone or in combination with chemotherapy. Initial phase I clinical trials of single and multiple dose weekly administration of $\mathrm{C} 225$ have shown that the antibody is safe and with predictable pharmacology, achieving optimal antibody serum levels for a prolonged period of time.

A new family of potent EGFR tyrosine kinase inhibitors (TKI) has been recently shown to have a high degree of receptor specificity and very potent antitumor activity in the laboratory. We are currently conducting a phase I clinical trial with ZD1839, a potent EGFR TKI, in patients with advanced malignancies. We have observed inhibition in vivo of receptor function by tumor and skin biopsies, and anti-responses have been observed.

\title{
S-14 Use of the anti HER-2/neu antibody Herceptin in the treatment of human breast cancer: biological rationale and clinical results
}

\author{
DJ Slamon \\ Division Hematology-Oncology, UCLA School of Medicine, Revlon/UCLA Women's Cancer Research Program, Jonsson \\ Comprehensive Cancer Center, Los Angeles, California, USA
}

The HER-2/neu proto-oncogene encodes a growth factor receptor which is overexpressed in 25-30\% of human breast cancers. This pathologic overexpression is associated with a decreased relapse-free as well as overall survival in those patients whose tumors contain the alteration. The overexpression is most often due to amplification in $~ 95 \%$ of cases. The association between HER-2/neu amplification/overexpression and clinical outcome suggested that the alteration may play a causal role in pathogenesis. To test the potential role of $H E R-2 / n e u$ overexpression in altering the biological activity of human breast normal and malignant epithelial cells, we conducted a number of in vitro studies in which single-copy, low-expressing cell lines were converted to multiple-copy, high-expressing cells. The biological effects of HER-2/neu overexpression were then measured, including effects on DNA synthesis, cell growth, anchorageindependent growth, tumorigenicity and metastatic potential. Overexpression of HER-2/neu resulted in an increase in those parameters in the malignant cell lines as well as the non-transformed immortalized breast cell lines. In normal primary breast cells there was no evidence of these effects with HER-2/neu overexpression alone.

We also tested the effects of HER-2/neu overexpression on chemosensitivity to a number of agents. There were no effects of overexpression on intrinsic sensitivity or resistance to any of nine chemotherapeutic agents, including anthracycline and taxanes. There were, however, effects on hormone dependence and tamoxifen sensitivity with a direct association between HER 2 overexpression and estrogen independence as well as tamoxifen resistance.
Subsequent to the identification of this alteration and demonstration of the role it plays in the pathogenesis of aggressive breast cancers, we tested a number of antibody reagents directed against the extracellular domain of this receptor from a variety of sources. Many of these antibodies can suppress all of the biological effects induced by HER-2/neu overexpression both in vitro and in vivo. Preclinical studies indicate that the antibodies can be effective in completely suppressing growth of human tumor cells in vitro, as well as breast cancer xenografts when either are growing in vivo. The suppression is specific to cells and tissues overexpressing the HER-2/neu gene. Strategies using anti HER-2/neu in combination with other therapeutic modalities indicates these antibodies can have additive and occasionally synergistic effects with chemotherapeutic agents both in vitro and in vivo. These observations have led to the development of new treatment strategies directed at this molecular alteration, and these strategies have completed clinical testing. The pivotal phase III study comparing best available standard therapy versus best available plus Herceptin demonstrates that this new biological agent improves objective response rates by $54 \%$, response duration by $58 \%$ and time to progression by $65 \%$. In addition, initial use of Herceptin as part of the combination therapy results in a decrease in relative risk of death by approximately $25 \%$ at two and one half years. The results of this testing have led to the approval of Herceptin, a therapeutic monoclonal antibody effective in HER-2/neu overexpressing breast cancers. 


\section{S-15 Herceptin in clinical use - early 2000}

\section{Vogel}

University of Miami School of Medicine, USA

Herceptin is the first of a new generation of non-cytotoxic, non-hormonal compounds showing considerable promise in the management of metastatic breast cancer. Based on the seminal work of Slamon and co-workers, the humanized monoclonal anti-Her-2-neu antibody entered clinical trials in the early 1990s. While the initial phase II clinical trial publication by Baselga and co-workers did not receive the kind of attention it should have been given, discovery of activity in heavily pre-treatment patients with the use of a relatively non-toxic antibody led to further trials establishing the effectiveness of this unique compound.

Two pivotal trials were initiated, with one investigating the use of Herceptin in patients failing one or two prior chemotherapy regimens for metastatic breast cancer. In this trial the response rate was $20 \%$ as assessed by the investi- gator and $14 \%$ by an external Response Evaluation Committee. Patients with 3+ Her-2-neu over-expression appeared to benefit more ( $17 \%$ vs $7 \%$ ) than Her-2-neu $2+$ over-expressors. Toxicity was mild with fever and chills seen in about $30 \%$ of patients, but generally only with the $4 \mathrm{mg} / \mathrm{kg}$ loading dose and seldom with the $2 \mathrm{mg} / \mathrm{kg}$ maintenance doses.

In the other pivotal trial, patients were randomized to chemotherapy alone or with Herceptin as first-line therapy for metastatic breast cancer. Women who had not received prior anthracycline therapy were randomized to anthracycline \pm Herceptin, while patients with prior adjuvant anthracycline were randomized to paclitaxel \pm Herceptin. Herceptin plus chemotherapy was superior to chemotherapy alone in all parameters of effectiveness, including a 5-month survival advantage at two years of follow-up.

\section{S-16 Characterisation of micrometastatic tumor cells}

\section{K Pantel}

Molecular Oncology, Department of Gynecology and Obstetrics, University Hospital Eppendorf, Hamburg, Germany

Using monoclonal antibodies to epithelial cytokeratins (CK) or tumor-associated cell membrane glycoproteins, individual carcinoma cells can be detected on cytological preparations at frequencies of $10^{-5}$ to $10^{-6}$ (for review, see Pantel et al, JNCl, 1999). Our prospective clinical studies have shown that the presence of these immunostained cells in bone marrow and lymph nodes of patients without clinical or histopathological signs of metastases is prognostically relevant (Pantel et al, Lancet, 1996; Izbicki et al, N Engl J Med, 1997; Braun et al, N Engl J Med, 2000). In addition to immunocytochemistry, new molecular detection methods based on the amplification of a marker mRNA species by the polymerase chain reaction technique have been developed (Zippelius et al, JCO, 1997). The current assays may be used to improve tumor staging with potential consequences for adjuvant therapy. Another promising clinical application is monitoring the response of micrometastatic cells to adjuvant therapies (Braun et al, JCO, 2000), which, at present, can only be assessed retrospectively after an extended period of clinical follow-up. Moreover, the screening methods can be applied to detect tumor cells in the autologous transplant. The extremely low frequency of bone marrow tumor cells greatly hampers approaches to obtain more specific information on their biological properties. The tools established in our laboratory (eg, micrometastatic cell lines, single cell (RT)PCR, multiple labelling, and FISH) allow one to obtain further insights into the phenotype and genotype of therapy-sensitive and resistant micrometastases. The available data indicate that micrometastatic cells represent a selected population of cancer cells which, however, still express a considerable degree of heterogeneity with regard to chromosomal aberrations and phenotypic properties. Prominent characteristics of bone marrow tumor cells at the time of primary tumor diagnosis are the lack of both p53 mutations and proliferation-associated marker proteins and the frequent overexpression of the erbB2 oncogene (Pantel et al, JNCl, 1993; Putz et al, Cancer Res, 1999; Offner et al, PNAS, 1999). Identification of the molecular determinants of micrometastasis may help to design new strategies to detect and eliminate minimal residual cancer.

\section{S-17 Animal models of human tumor suppressor genes}

\section{TW Mak}

Ontario Cancer Institute, Canada

Tumor suppressor genes are a class of genes found mutated on both alleles in tumor cells. They are usually implicated in DNA repair, cell-cycle progression, differentiation, and apoptosis. Their loss of function is involved in the development of malignancies. Many of these tumor suppressor genes are also found mutated in familial cancers. To better understand the development of tumors that have lost the functions of these tumor suppressor genes, we have generated animal models by targeting these genes in the germline of mice. Many of these mutations, when bred to homozygos- 
ity, however, are embryonic lethals in the mice. Consequently, conditional knockouts of these genes are used to study their functions. They include tissue-specific deletions and inducible knockouts. Progress in these genetic manipulations to study tumor development in animals with mutations of BRCA1, DPC4, and PTEN will be described.

S-18 Drug discovery in the p53 pathway

\section{DP Lane, C Midgley, A Sparks, C Blattner, C Binden and S Laine}

CRC Laboratories, University of Dundee, Dundee, UK

The activity of the tumour suppressor protein p53 is critically controlled by proteolysis. When cells are exposed to a variety of stress stimuli including hypoxia, DNA damage, or the action of certain oncogenes, this degradative pathway is inhibited and p53 protein levels rise, inducing cell-cycle arrest and apoptosis. The function of the p53 pathway is affected by many DNA tumour virus-derived oncogenes. In addition, two cellular proteins, Mdm2 and Arf, have been discovered to play a critical role in regulating the specific stability of p53. Mdm2 binds to the $N$ terminus of $p 53$, recognising a specific peptide motif, and targets p53 to the proteasome. The Mdm2 protein acts as a specific E3 ubiquitin ligase, and the Arf protein binds to Mdm2 and inhibits its ligase activity. Recently we have also discovered that p53 is modified by the small ubiquitin-like protein SUMO, and this modification may inhibit the degradation of p53. Microinjection of antibodies to the p53-binding domain of Mdm2 will activate the p53 response in normal cells as will mini-proteins displaying phage-opti- mised Mdm2 binding peptides that block p53 binding. The $\mathrm{N}$ terminal 64 amino acids of Arf are also a potent activator of the p53 response, and we have recently localised the Arf-Mdm2 interaction using pepscan libraries of Arf. An important feedback pathway exists because the Mdm2 gene is only transcribed in cells that contain normal p53.This explains why tumours stain strongly with anti-p53 antibodies if that p53 is inactive as a transcription factor. In a survey of small molecules we found that both the nuclear export inhibitor leptomycin B and the proteasome inhibitor Lactacystin caused the accumulation of p53 in normal cells. Strikingly, only Leptomycin-induced p53 was transcriptionally active. Using inducible cell-line systems, we established that Mdm2 targets p53 for nuclear export. The ability to induce the p53 response with non-genotoxic agents combined with the recognition that p53 mutant human tumours lack the Mdm2 dependant degradation pathway opens up many exciting new approaches to drug discovery in the p53 pathway.

\section{S-19 Carcinoma-associated fibroblasts stimulate tumor progression of initiated human epithelium}

AF Olumi, GD Grossfeld, SW Hayward, PR Carroll, GR Cunha, P Hein and TD TIsty

Cancer Research Center, Departments of Urology, Pathology and Anatomy, University of California at San Francisco,

San Francisco, USA

We have demonstrated that fibroblasts associated with carcinomas stimulate tumor progression of initiated nontumorigenic epithelial cells both in an in vivo tissue recombination system and in an in vitro co-culture system. Human carcinoma-associated fibroblasts grown with initiated human epithelial cells dramatically stimulate growth and alter histology of epithelial cells. This effect is not detected when normal fibroblasts are grown with the same epithelial cells under the same experimental conditions. From these data and other data we conclude that, in this human cancer model, carcinoma-associated fibroblasts stimulate tumor progression of an initiated epithelial cell.

\section{p53-mediated apoptosis and genomic instability syndromes}

EA Spillare, XW Wang, SP Linke, A Robles and CC Harris

Laboratory of Human Carcinogenesis, National Cancer Institute, NIH, Bethesda, MD, USA

The eukaryote genome is constantly facing the threat of damage from exogenous and endogenous mutagens. Mammalian cells, therefore, have evolved an intricate network of defenses to maintain genomic stability, eg, cellcycle checkpoints, DNA repair, and apoptosis. Defects in these processes can result in a mutator phenotype associated with tumorigenesis, as exemplified by a number of familial cancer-prone disorders, including xeroderma pigmentosum (XP), Bloom syndrome (BS), ataxia telangiectasia (AT), Werner syndrome (WS) and Li-Fraumeni syndrome (LFS). p53 is at the crossroads of these pathways, and provides a biological basis for p53 being a prime target of somatic mutations in human cancers. We are investigating the molecular mechanisms related to these pathways. For example, p53 binds to the basal transcription and nucleotide-excision repair complex, TFIIH, through interaction with two DNA helicases, XPB and $X P D$, and cells with p53 inactivation have a reduced DNA repair activity. Using a genetic approach, we also showed that XPB and XPD contribute to p53-mediated apoptosis. These data indicate that p53 may modulate either DNA repair or apoptosis by binding to and regulating the activity of the TFIIH-associated DNA helicases. We are also investigating the physical and functional interactions between p53 and other DNA helicases, including WRN and BLM. Our data are consistent with the hypothesis that 
WRN and BLM contribute to the removal of blocks in DNA replication due to either errors during DNA metabolism or carcinogen-induced DNA damage. WS or BS fibroblasts have an attenuated p53-mediated apoptotic response, and this deficiency can be rescued by the expression of wild-type WRN or BLM, respectively. These data further support the hypothesis that p53 can induce apoptosis through the modulation of specific DExH-containing DNA helicases, and may have implications for the cancer predisposition observed in these genomic instability diseases.

\section{Lessons from TP53 mutations in breast cancers: from carcinogen fingerprints to clinical correlates}

P Hainaut and M Olivier

International Agency for Research on Cancer, Lyon, France

About 1000 mutations in breast cancers are listed in the IARC TP53 mutation database [1]. Overall, the mutation prevalence is relatively low (20-30\%). Mutations are associated with most aggressive tumor types and carry a significant risk of bad prognosis and outcome in both node-positive and node-negative tumors. Among tumors expressing mutant p53, those with mutations in the L2/L3 loops of the protein (DNA-binding surface) have a poorer response to some forms of treatment than tumors with mutations at other sites [2]. It is noteworthy that p53 protein levels are elevated in more than $50 \%$ of breast cancers, suggesting that p53 function may be deregulated by mechanisms other than mutation.

The pattern of TP53 mutations shows a relatively high prevalence of insertions, deletions and nonsense mutations (altogether, 25\%). The most frequent mutation type is GC to AT transitions (40\%), equally affecting $\mathrm{CpG}$ and non$\mathrm{CpG}$ sites. Cohort comparisons have shown differences in the nature, localization and frequency of mutations, but these studies need to be substantiated on larger groups [3].

Breast cancer frequently arises in Li-Fraumeni families [4]. The mutations found in this context may be considered as representative of spontaneous mutations arising in breast cancer. Comparison with sporadic cancer shows that two transversions, $\mathrm{G}$ to $\mathrm{T}$ and $\mathrm{G}$ to $\mathrm{C}$, are not found in Li-Fraumeni breast cancer patients. These transversions represent $18 \%$ of somatic breast-cancer mutations. They show a strong strand bias and occur at sites often mutated in lung cancers from smokers (codons 157, 248, 249 and 273) or in bladder cancers from smokers and/or dyeexposed workers (codons 158 and 280). Overall, these data indicate that although most of breast cancer mutations probably have a spontaneous origin, a small proportion of mutations show signatures that suggest the involvement of exogenous carcinogens.

\section{References}

1. Hernandez-Boussard T, Rodriguez-Tome $P$, Montesano R, Hainaut $P$ : Hum Mutat 1999, 14:1-8.

2. Aas $T$, Børresen $A L$, Geisler $S$, Smith-Sorensen $B$, Johnsen $H$ Varhaug JE, Akslen LA, Lonning PE: Nature Med 1996, 2:811-814.

3. Blaszyk H, Hartmann A, Sommer SS, Kovach JS: Hum Genet 1996, 97:543-547.

4. Ohgaki $\mathrm{H}$, Hernandez $\mathrm{T}$, Kleihues $\mathrm{P}$, Hainaut $\mathrm{P}$ : In Molecular Biology and Cancer Medicine, 2nd Edition, edited by Kurzock R and Talpaz M. Martin Dunitz, 1999:477-492.

\section{S-22 p53 action in apoptosis and senescence}

SW Lowe, CA Schmitt, G Ferbeyre, AW Lin, E de Stanchina and M Soengas

Cold Spring Harbor Laboratory, Cold Spring Harbor, NY 11724, USA

Our laboratory is interested in the genes that control apoptosis and cellular senescence, two conceptually related processes that can act to limit cellular proliferation. Both processes are frequently disrupted in cancer cells, implying that each can limit tumor development. Moreover, since radiation and many chemotherapeutic agents can activate apoptosis or senescence, the integrity of these anti-proliferative programs may influence the outcome of cancer therapy in patients. The p53 tumor suppressor can promote apoptosis or senescence and, together with its cell-cycle checkpoint function, acts at in a variety of ways to protect against cancer. For example, p53 can be activated by DNA damage to activate cell-cycle checkpoints or apoptosis, such that cells lacking p53 are prone to certain forms of mutation and genomic instability. This implies that p53 can indirectly suppress tumorigenesis by acting as a 'Guardian of the Genome', that is, to promote the repair or elimination of cells sustaining potentially deleterious mutations. Remarkably, since most current anticancer agents directly or indirectly damage DNA, the integrity of this p53 response may contribute to tumor cell death during therapy. In addition, certain mitogenic oncogenes activate p53 to promote apoptosis or senescence. Loss of p53 prevents these responses, leading to oncogenic transformation or tumor progression. In these settings, p53 can directly suppress tumorigenesis by acting in a fail-safe mechanism to counter hyperproliferative signals. We are currently studying many aspects of $p 53$, including how oncogenes or DNA-damaging agents activate p53, how p53 executes a biological response, and how cellular factors influence whether p53 induces a cell-cycle checkpoint, cellular senescence, or apoptosis. We are also developing animal models to examine the impact of the p53 pathway on tumor cell responses to anticancer agents. 


\section{S-23 Cancer susceptibility in ATM heterozygotes: do two distinct carrier populations exist?}

RA Gatti ${ }^{*}$ and P Concannon ${ }^{+}$

*UCLA School of Medicine, Department of Pathology, Los Angeles, CA, 'Virginia Mason Research Center, Seattle, WA, USA

The failure to find an increased frequency of ATM mutations in large cancer cohorts, especially breast cancer, is contrary to what was anticipated based on the increased cancer susceptibility of obligate ATM heterozygotes from families with ataxia telangiectasia. This apparent contradiction might be resolved if two types of ATM heterozygotes were to exist and their phenotypes were to differ, ie, those with truncating types of mutations (ATMirunc) that make no protein, and those with missense types of mutations $\left(\right.$ ATM $\left.^{\text {mis }}\right)$ that make reduced amounts of defective protein; the latter could create a dominant negative effect that could be more detrimental than having no protein at all. The phenotype of $A T M^{\text {trunc/trunc }}$ mutations is the AT syndrome; the phenotype of $A T M^{\text {mis/mis }}$ mutations, judging from the few homozygous patients that have been documented, appears to include some neurological features and cancer susceptibility but not the typical AT syndrome. Evidence will be presented which suggests that ATM ${ }^{\text {mis/wt }}$ mutations are technically more difficult to detect than ATM ${ }^{\text {trunc/wt }}$ mutations. Despite this, most large cancer cohort studies have identified mainly missense mutations and few truncating mutations. If substantiated, this model would require a paradigm shift for cancer risk analyses that would recognize the existence of different allelic frequencies for the missense and truncating ATM heterozygotes.

S-24 Role of the ATM gene in radiation sensitivity, relevance to breast cancer treatment

J Hall*, S Angèle*, M Vuillaume*, J-P Gérard† and P Romestaing ${ }^{\dagger}$

*International Agency for Research on Cancer, Lyon, ${ }^{+}$Service de Radiothérapie-Oncologie, Centre Hospitalier Lyon Sud,

Pierre Bénite, France

Clinical observations of normal tissue damage are observed in a subset of patients following radiotherapy, with several studies reporting that up to $10 \%$ of breast cancer patients show early or late tissue reactions. Mutations in the Ataxia telangiectasia gene (ATM) result in extreme radiation sensitivity: homozygotes are predisposed to developing cancers at a young age and show an acute radiation reaction when treated with conventional radiotherapeutic doses for cancer. Heterozygotes have an increased cancer risk, in particular breast cancer, and some degree of sensitivity to ionising radiation (IR) has been reported in in vitro studies. To evaluate the potential role of the ATM gene in breast cancer development and the radiosensitivity seen in certain breast cancer cases, we have established lymphoblastoid cell lines (LCL) from radiosensitive (EORTC $>3$ ) and non-radiosensitive breast cancer patients. In some of the LCLs established from radiation sensitive breast cancer patients, the level of cell survival and the p53 induction after IR exposure are lower than those observed in control cell lines, indicative of an alteration in the ATM signalling pathway. No ATM mutations were detected in the LCLs from the 10 non-radiosensitive breast cancer patients, whereas one truncating mutation and 3 nucleotide changes were found in 4 out of 27 LCLs from the radiosensitive patients. The frequency of these nucleotide alterations in the general population is being established, in order to determine whether they represent cancer and/or radiation sensitivity predisposing mutations.

\section{Acknowledgements}

Financial support to $\mathrm{JH}$ from the Association pour la Recherche sur le Cancer and La Ligue Nationale Contre le Cancer, Comité Départemental du Rhône is gratefully acknowledged.

\section{S-25 Tumour-selective transcriptional targeting for breast cancer gene therapy}

\section{HC Hurst}

Imperial Cancer Research Fund Molecular Oncology Unit, ICSM at Hammersmith Hospital, London W12 ONN, UK

One of the most attractive ways of targeting gene therapy is by exploitation of the transcriptional regulatory elements of genes which display tissue- or tumourselective patterns of expression. In our Unit we have focused on identifying the key regulatory elements in a small number of genes known to be overexpressed in breast tumours. Shortly, however, the power of expression profiling by chip technology will expand the range of candidate genes enormously. This will also allow much more specific expression-targeting constructs to be developed by 'mixing and matching' elements from different genes.
Genetic prodrug activation therapy (GPAT) depends on the conditional expression of a gene encoding an enzyme capable of converting a non-toxic prodrug into an active cytotoxic agent. We have developed prototype systems based on the proximal promoter of the human ERBB2 oncogene driving a variety of suicide genes in plasmid, retroviral and adenoviral vectors. We have completed a phase 1 clinical trial of direct intratumoral injection of an ERBB2-cytosine deaminase plasmid in patients with advanced breast cancer, and the system is about to be applied to other tumour types. Tissue-selective targeting has also been explored using the promoter and enhancer elements of the MUC1 gene, char- 
acteristically expressed by simple ductal epithelial cells including those of the breast and pancreas. Combination of the MUC1 and ERBB2 elements has proved effective in generating constructs with dual specificity targeting. In addition, the range of suicide genes which can be delivered with such targeting devices can be expanded by the use of high capacity adenoviral vectors, which allow combinations of suicide genes to be expressed and thus increase therapeutic effect without loss of selectivity.

\title{
S-26 G1/S control and its deregulation in cancer
}

J Bartek, C Lukas, C Sørensen, E Santoni-Rugiu, J Bartkova and J Lukas

Institute of Cancer Biology, Danish Cancer Society, Copenhagen, Denmark

Cancer is increasingly viewed as a cell-cycle disease, a notion supported by recent accumulation of data on the molecular basis of the cell-cycle machinery and its defects commonly found in human tumours including breast carcinomas. Strikingly, the cell-cycle phase targeted most frequently in multistep oncogenesis is the control of $\mathrm{G} 1 / \mathrm{S}$ transition. This period includes the late-G1 commitment to replicate the genome and complete the cycle (the restriction point control), and the initiation of DNA replication, events regulated by the so-called 'RB pathway'. While the key components of the RB pathway qualify as proto-oncogenes or tumour suppressors, and their aberrations may provide direct proliferative advantage to cancer cells, defects in the so-called checkpoint mechanisms that monitor and help ensure the error-free execution of the cell-cycle transitions act more indirectly, yet affect both tumour progression and response to anti-cancer therapy.
Examples of both the oncogenic defects in the G1/S-controlling machinery, and the ways proto-oncogenic events may activate checkpoint responses, will be presented. In addition, evidence in favour of the existence of a parallel pathway, independent of and cooperating with the classical p16-cyclin D/CDK-pRB-E2F axis (the RB pathway) to govern timely S-phase entry, will be reported. Finally, the proposed candidacy of the RB pathway for the molecular mechanism underlying the late-G1 restriction point switch will be critically considered, and emerging data on novel functions of the RB pathway in coordination of the cell cycle events from late $\mathrm{G} 1$ until mitosis will be summarized. These new discoveries have significant implications for our understanding of the mammalian cell-cycle control and its subversion in tumour cells, with emerging applications in tumour diagnosis, prognosis, and attempts to device new strategies to treat cancer.

\section{S-27 Molecular control of apoptosis in the breast}

\author{
C Streuli, A Gilmore, A Metcalfe, Y-J Lee, J Oliver and A Valentijn \\ School of Biological Sciences, University of Manchester, Manchester, UK
}

Understanding the molecular control of apoptosis in breast epithelium represents an exciting new challenge in breast biology. As a prerequisite for unravelling potential mechanisms for apoptotic defects in neoplasia of the breast, we have opted to decipher its regulation in normal mammary epithelium. Apoptosis occurs naturally at several stages of breast development; during the formation of intraductal lumina, at the end of each menstrual/oestrus cycle, and during involution that follows lactation. In the latter case, experimental manipulation of nursing can result in massive and synchronised epithelial cell apoptosis. Moreover, the culture of primary breast epithelial explants can be manipulated to allow synchronous apoptosis. We have used these experimental systems to define both extracellular regulators of survival and the intracellular components of the Bcl-2 family that are involved with apoptotic decisions in mammary epithelium.

It is well known that soluble factors are essential for cell survival, and both EGF and insulin act to suppress apoptosis in mammary epithelium. It has also become clear that adherent epithelial cells require interactions with the extra- cellular matrix for their survival. Indeed, interplay between these two types of extracellular survival factor occurs at the level of intracellular signal transduction. We shall show that the specialised ECM known as basement membrane controls the ability of insulin to deliver PI 3-kinase-regulated survival signals, and that this is mediated through the integrin class of receptors.

Commitment to mammary apoptosis in vivo is regulated through a wide spectrum of Bcl-2 family proteins, including Bcl-x, Bcl-w, Bax, Bak, and Bad. Part of the control of apoptosis comes from the developmental regulation of gene expression in this family, since transcription of Bcl-w, Bak and Bad is altered as cells enter post-lactational involution. In addition, cell-autonomous regulation of apoptosis in the absence of protein synthesis is also a key player, and the cell death protein Bax regulates apoptosis through its subcellular localisation. In healthy cells Bax is cytoplasmic, but altered survival signalling promotes translocation to mitochondria: we now know that the ECM has a critical role in regulating Bax distribution, and that this occurs through protein tyrosine kinase signal-transduction pathways. 


\section{S-28 Hypoxia and tumour angiogenesis involved in breast cancer progression and targets for therapy}

AL Harris

ICRF Medical Oncology Unit, Churchill Hospital, Oxford, OX3 7LJ, UK

Tumours cannot grow above 1-2 $\mathrm{mm}$ in diameter without developing a new blood supply. We, and others, have shown that the number of blood vessels in breast cancer is related to prognosis, and that multiple growth-factor pathways control angiogenesis. One of the most important ones is vascular endothelial growth factor and thymidine phosphorylase. Both are targets of therapy, and currently there are trials running inhibiting VEGF signalling pathway and also using prodrugs activated by thymidine phosphorylase to produce cytotoxic agents. Hypoxia is a major factor switching on angiogenesis, and new approaches using the hypoxia signalling pathways to activate gene therapy in breast cancer have been initiated. Hypoxia activates vascular endothelial growth factor production by the transcription factor hypoxia inducible factor 1a (hif1), which binds to specific DNA sequences. Using DNA constructs with multimers of these response elements has generated reagents that will specifically switch on under hypoxia and activate prodrugs. Since tumours are much more hypoxic than normal tissues, this should provide marked differential expression. Also, these areas of tumours are resistant to radiotherapy, so it will be particularly useful in worse prognosis resistant tumours. We raised antibodies to hif1, and to another member of the family, hif2.

Staining breast tumours showed marked induction of hif2 expression in stromal macrophages, whereas hif1 was expressed in the epithelium around the areas of necrosis. Thus, there are two different hypoxia-regulated pathways activated. We have also screened for novel genes induced by hypoxia, and found that another transcription-factor pathway downstream of hif as well as enzymes regulating $\mathrm{pH}$ are modified. These are new potential targets for therapy.

\section{Telomerase: a breast cancer chemopreventive and therapeutic target}

\section{JW Shay}

The University of Texas Southwestern Medical Center, Dallas, Texas, USA

Telomeres are repetitive DNA sequences at the end of linear chromosomes. Each time a cell divides some telomeric sequences are lost. When telomeres are short, cells enter an irreversible growth arrest state called replicative senescence. In most instances cells become senescent before they can become a cancer cell. However, almost all cancer cells are immortal, having overcome cellular senescence. Maintenance of telomere stability is required for cells to escape from replicative senescence and proliferate indefinitely. Telomerase, a cellular reverse transcriptase, is upregulated or reactivated in most cancers and helps to stabilize telomere length by adding TTAGGG repeats onto the telomeres. The correlation between telomerase activity and human tumors suggests that tumor growth requires reactivation of telomerase and that telomerase inhibitors represent a novel class of chemotherapeutic agents. Human cancer cells treated with oligonucleotide-based antisense chemistries (peptide nucleic acid or 2'-OmeRNA), directed against the template region of telom- erase RNA, inhibit telomerase activity inside cells at pharmacological dosages. Telomerase inhibition leads to progressive telomere shortening, causing immortal human breast epithelial cells to undergo apoptosis. Telomerase is also being considered as a target for molecular chemopreventive strategies to inhibit immortalization. Expressing a dominant-negative mutant of the telomerase catalytic subunit prevents the spontaneous immortalization of TP53heterozygous Li-Fraumeni Syndrome-derived breast epithelial cells. These results not only validate telomerase as a target for breast cancer prevention and therapy, but also supply insights into the properties that successful antitelomerase agents will require. To confirm action through a telomerase-dependent mechanism, inhibitors but not chemically-related molecules should (i) reduce telomerase activity but not initially affect cell growth rates; (ii) lead to progressive shortening of telomeres with each cell division; and (iii) cause cells to die or undergo growth arrest in a time frame dependent on initial telomere length.

\section{S-30 Blood assays for earlier detection of cancers}

\section{AB Pardee, RJ Guan, HL Ford, MV Fournier, $K$ Martin and R Sager}

Dana-Farber Cancer Institute, Boston, MA, USA

A significant way to improve therapy against cancer is to detect cancer earlier than we can at present. Current methods are similar to trying to put out a fire after the house is in flames. Rather, we need to invent 'smoke detector' techniques that signal very early cancer or the

development of metastases. These would allow treatment of far fewer cancer cells, prior to extensive metastases and drug resistance. They should aid present treatments by surgery, radiation, chemotherapy and perhaps immunology and differentiation therapy. 
Our goal is to devise early warning systems. We already have found many mRNAs whose expression is modified in cancers (Sager, 1997; Ford, 1998; Fournier, 1999a, b; Guan, 2000; Martin, 2000). We are now developing markers and methods to detect very low numbers of tumor cells that are released into blood early in the progression of solid tumors. Some of these markers are found in $3 \mathrm{ml}$ blood samples from cancer patients, and are missing from blood of normal individuals.

To find new genes we apply the concept of Expression Genetics (Sager, 1997), using the differential display method (Pardee, 1999). We are analysing 200 differentially expressed mRNAs from breast cancers, applying high density membrane-based hybridization arrays. Cluster analysis identifies groups of genes whose expression patterns correlate with clinical information, including estrogen receptor status, tumor size, and stage (Martin, 2000). The early detection methods we are developing could also be applied to predict optimal cancer therapy, to determine therapeutic efficacy by measuring decreases of markers in blood, and to detect recurrence after therapy. These techniques could be applied to other body fluids.

\section{s-31 Abstract not submitted for publication}

\section{S-32 Predictive factors in breast cancer}

J Klijn, E Berns, L Dorssers and J Foekens

Dr Daniel den Hoed Cancer Center/Academic Hospital Rotterdam, The Netherlands

In the processes of malignant transformation, tumor cell growth regulation, angiogenesis and metastasis, and the development of drug resistance, a large number of oncogenes, suppressor genes, proteases and their inhibitors are involved. In addition to the classical clinical prognostic factors, many of these molecular biological parameters are increasingly used as prognosticator and predictive factors for response to therapy, and recently also as targets for new biologic therapeutic strategies.

In nearly 2800 patients with primary breast cancer and in 830 patients with recurrent disease we investigated by univariate and multivariate analysis the prognostic and/or predictive value of a large series of more than 20 molecular biological factors. In patients with primary breast cancer cmyc amplification, TP53 mutation and increased expression of cathepsins and/or components of the urokinase-type plasminogen (UPA) activator system showed clear prognostic value. The most powerful predictive factors for a favorable response to endocrine therapy are $\mathrm{ER}, \mathrm{PgR}$, and PS2, while HER2-neu, EGF-R, TP53 mutation and expression, uPA and TK are associated with a poor response to tamoxifen. Mainly TP53 mutations, TS and MRP expression were associated with type of response to chemotherapy. Germ-line mutations in the breast cancer genes 1 and 2 (BRCA1/2) were associated with a similar prognosis as observed in patients with sporadic breast tumors in spite of different tumor characteristics (Verhoog et al, Lancet 1998 and J Clin Oncol 1999). Recently a new gene associated with tamoxifen resistance, located at human chromosome 16q, was detected in over 97 patients. High Bcra1/p130Cas protein levels were associated with poor prognosis (ES van der Flier et al, J Natl Cancer Inst 2000, 92:120-127).

In conclusion, an increasing number of cell biological factors such as HER2-neu appear to be of clinical importance because of their predictive value and their role as (potential) targets for therapy.

\section{S-33 Clinical trials in breast cancer; do we have the right approaches?}

\section{PE Lønning}

Section of Oncology, Department of Medicine, Haukeland University Hospital, Bergen, Norway

While adjuvant chemotherapy as well as hormone therapy improve long-term survival in breast cancer patients [1,2], only about $25-30 \%$ of patients at risk are cured by such treatment modalities. While improved regimens using more effective chemotherapeutics have been shown to improve outcome marginally [3], the disappointing results from highdose chemotherapy with stem cell support $[4,5]$ underlines the limitations associated with current chemotherapy.

While expression of the estrogen and progesterone receptors have been used to select patients for endocrine treatment, so far we have lacked predictive factors with respect to outcome in chemotherapy. Over the last decade, laboratory investigations have revealed several potential mechanisms explaining resistance to chemotherapy. Thus, there is evidence that loss of function of the TP53 gene may confer resistance to chemotherapeutics like the anthracyclines but does not deteriorate response to the taxanes [6,7], a finding supported by recent studies in breast cancer patients $[8,9]$.

These findings may challenge the way we are running clinical trials in breast cancer patients. If certain gene mutations predict for resistance to specific drugs, the key target for future studies should be to outline these mechanisms in vivo. While combined therapy regimens may 
improve response rates to some extent, such approaches would imply over-treatment with increased toxicity in many patients who would not benefit from one or more of the drugs in the 'cocktail'. It may even be detrimental to clinical outcome as it may require reduction in the dose of active drugs. Retrospective evaluation of predictive factors in adjuvant studies are complicated by several confounding factors like inappropriate tissue sampling (paraffinembedded only), use of combined regimens and inferior surrogate markers for therapeutic efficacy (time to relapse versus direct antitumour effects). Evaluation of predictive factors should preferably be done in relation to monotherapy with single chemotherapeutics in the advanced or neoadjuvant setting, and such results are likely to have a strong influence on how we design adjuvant studies in the future.

\section{References}

1. Abe O et al: Lancet 1998, 352:930-942

2. Clarke $\mathrm{M}$ et al: Lancet 1998, 351:1451-467.

3. Levine MN et al: J Clin Oncol 1998, 16:2651-2658.

4. Peters W et al: Proc Am Soc Clin Oncol 1999, 18:Abstr, 2, p.1a.

5. Scandinavian Breast Cancer Group Study: Proc Am Soc Clin Oncol 1999, 18: Abstr. 3, p.2a.

6. Lowe $S$ et al: Science 1994, 266:807-810.

7. Wahl AF et al: Nature Med 1996, 2:72-79.

8. Aas T et al: Nature Med 1996, 2:811-814.

9. Kandioler-Eckerberger D et al: Proc Am Soc Clin Oncol 1998, 17: 102a (Abstr. 392).

\section{Tissue microarrays for high-throughput profiling in molecular oncology}

\section{O-P Kallioniemi, J Kononen and G Sauter}

Cancer Genetics Branch, National Human Genome Research Institute, National Institutes of Health, Bethesda, MD 20852, USA and Institute of Pathology, University of Basel, Switzerland

High-throughput genome screening technologies, such as CGH, cDNA microarrays, SAGE, differential display, and DNA sequencing have made it possible to survey thousands of genes per tumor. The translation of such information to improved diagnostic, prognostic and therapeutic applications in the clinic requires extensive data 'mining' as well as validation, prioritization and extension of such results to hundreds or thousands of clinical specimens. This is often extremely tedious with conventional molecular pathology technologies. We have developed a novel technology, tissue microarrays ('tissue chips') for facilitating such 'genome-scale' translational cancer research [1]. This technology enables high-throughput molecular analyses of hundreds of tissue specimens or cells in a single experiment. Tissue microarrays are constructed by acquiring cylindrical biopsies from 500-1000 individual tumor tissues into a tissue microarray block, which is then sliced to over 200 sections for probing DNA, RNA or protein targets. A single immunostaining or in situ hybridization reaction now provides information on all of the specimens on the slide, while subsequent sections can be analysed with other probes or antibodies. Construction of multiple replicate blocks may allow up to 100000 sections to be generated from the same series of tumor specimens. This expands the scope of microarray technologies to the rapid, very large-scale molecular analysis of thousands of tissue specimens with thousands of probes for various DNA, RNA and protein targets. For example, we have utilized the combination of cDNA and tissue microarray technologies to uncover genes involved in breast and prostate cancer progression. In summary, tissue microarrays provide a powerful approach for the in vivo validation of gene discoveries, as well as a means to rapidly assess the clinical significance of molecular alterations.

\section{Reference}

1. Kononen et al: Nature Med 1998, 4:844-847.

\section{Two molecular cytogenetic views of breast cancer}

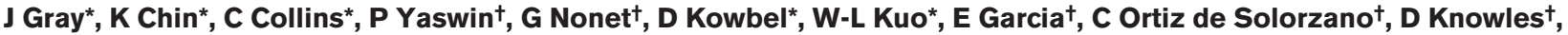 S Lockett ${ }^{\dagger}$, M Bissell $^{\dagger}$, V Weaver ${ }^{\dagger}$, D Pinkel*, D Albertson*, A-L Børresen-Dale ${ }^{\ddagger}$ and F Waldnian* \\ *UCSF Cancer Center, University of California, San Francisco; ${ }^{\dagger}$ Biomedical Sciences Division, Lawrence Berkeley National Laboratory, Berkeley, CA, USA and ¥Institute for Cancer Research, The Norwegian Radium Hospital, Oslo, Norway}

This talk will present two views of genome evolution in human breast cancers using fluorescence in situ hybridization (FISH) and comparative genomic hybridization (CGH). FISH with chromosome-specific probes applied to thick tissue sections from tumors at several stages of progression reveals a remarkable level of cell-to-cell variability beginning with hyperplasia and increasing with increasing grade. These studies also demonstrate significant genomic evolution, including formation of polyploid nuclei within a single sample, suggesting a very high rate of genomic instability. These phenomena also were observed in vitro in few-cell clones established from breast cancer cell lines. Paradoxically, CGH analyses of 'average' genome copy number changes show relatively slow overall rates of evolution in vitro and in vivo. CGH karyotypes for breast cancer cell lines change relatively slowly as long as the environment remains constant. Likewise, CGH karyotypes of pairs of primary vs. metastatic or in situ vs. invasive breast tumors from the same patient are usually quite similar, suggesting a relatively slow rate of evolution. Possible explanations for this paradox will be discussed. 
Higher resolution analyses of selected regions of recurrent genomic abnormality on chromosome 20 , using array $\mathrm{CGH}$ and $\mathrm{FISH}$, suggest the coordinate amplification of several genes that play a role in breast cancer evolution. Complete sequencing of $\mathrm{a} \sim 1 \mathrm{Mb}$ wide region centered on a region of recurrent amplification at $20 \mathrm{q} 13.2$ has revealed several genes that appear as possible candidate 'driver' genes including ZNF217, a gene now implicated in the immortalization of breast epithelial cells.

\section{S-36 Studying breast cancer on a genomic scale using DNA microarrays}

\section{Botstein and PO Brown}

Stanford University School of Medicine, Stanford, California, USA

Genome-wide studies of human gene expression have become possible in recent years because of the availability of most of the sequences of protein-encoding genes. Technology has been developed in our group to obtain and analyze patterns of expression of many thousands of genes at once. We have begun to apply cDNA microarray technology to the study of cancer, focusing on breast cancer. We characterized the variation in patterns of gene expression in a set of 62 surgical specimens of human breast tumors from 40 different patients, using cDNA microarrays representing 8102 different human genes. The observed gene expression patterns provided a remarkably distinctive molecular portrait of each tumor. Twenty of the tumors had been sampled twice, both before and after a 16-week course of doxorubicin chemotherapy, and two tumors were paired with a lymph node metastasis from the same patient. The gene expres- sion patterns observed in the serial samples from the same tumor, and the tumor/metastasis pairs, were almost always more similar to each other than either was to any other samples. Clusters of co-expressed genes were identified, for which variation in mRNA levels could be related to specific features of physiological variation, or to variation in the cellular constituents of the tumors. The tumors could be classified into subtypes that were distinguished by pervasive differences in their gene expression patterns. Such classifications were robust, and reflected changes in gene expression in the epithelial cells as well as differences in expression derived from the populations of nonepithelial cells in the tumors. These results suggest that gene expression patterns can provide distinctive and recognizable molecular portraits of individual tumors, and perhaps a basis for a new molecular classification of cancers. 


\section{Poster presentations}

\section{PI-01 Denaturing high-performance liquid chromatography (DHPLC) as a fast tool to screen a control population for the occurrence of unclassified variants (UV) in the BRCA1 gene}

N Arnold, E Gross, H Peper, K Bandick, W Jonat and M Kiechle

Department of Gynecology and Obstetrics, Michaelisstr. 16, 24105 Kiel, Germany

The identification of BRCA1 and BRCA2 mutations has enabled physicians to identify persons at high risk for carcinoma of the breast and ovary in hereditary breast-ovarian cancer (HBOC) families. Tests for known mutations are very sensitive and specific. The interpretation of previously undescribed variants (UV) is complicated because it could be either a cancer-causing mutation or a polymorphism. Until a functional test is available, general population frequency analysis of unclassified variants in the BRCA1 coding region is useful to support the putative role of missense mutations. In contrast to the very cumbersome evaluation of sequence data, the evaluation of results by DHPLC is quite effortless because the investigator has to discriminate only between single and multiple peaks in the elution profiles. The following reported UVs were analysed with the DHPLC technique: A835G; C1605T; G2531C; A2577G; C2596A; C2640T;
C2715T; G3238A; G4654T; T5002C; C5029T; G5075A and 5136 del CAC. The analysis was done with DNA from 98 persons ( 81 females and 17 males) who had no BRCA1 related carcinomas in their family history. The ages for the females range from 58 to 92 years (median 73 years) and for the males from 58 to 87 years (median 70 years). With this strategy we detected G5075A four times, G3238A two times and C2715T one time. Therefore, these BRCA1 alterations can be considered as rare polymorphisms. The other investigated UVs could not be detected in the samples and the nature of the alterations still remains unclear. With the DHPLC technique we also detected two novel alterations G1606A and G1736A. Sequence comparison with dog $B R C A 1$ reveals that $\mathrm{G} 1606 \mathrm{~A}$ represents a wild-type constitution and $\mathrm{G} 1736 \mathrm{~A}$ is a silent mutation. Therefore both alterations represent rare polymorphisms.

PI-02 Clinical and pathological characteristics in patients with BRCA1/2-mutation associated with breast cancer (BC) with a long clinical follow-up

O Díez*, C Pericay†, M Domènech*, J Brunet ${ }^{\dagger}$, J Cortés*, J Balmaña†, A Gómez ${ }^{\ddagger}$, JJ López ${ }^{\dagger}$, MC Alonso ${ }^{\dagger}$ and M Baiget*

*Serveis de Genètica, ${ }^{\dagger}$ Oncologia Mèdica y ${ }^{\ddagger}$ Radiodiagnòstic, Hospital de Sant Pau, Barcelona, Spain.

It is not known if the behaviour of hereditary breast cancer (HBC) differs from that of sporadic BC. We analysed clinico-pathological characteristics in 17 patients with $B R C A 1 / 2$ germline mutations associated with $B C$. These data could be useful in the management of HBC. All patients except 4 had a family history of $B C$ or ovarian cancer (OC). Mutation detection methods were SSCP and PTT (exon 11 for BRCA1, exons 10 and 11 for $B R C A 2)$ and final direct sequencing. The BRCA1 mutations found were: exon 2 185delAG, exon 2 189insTGTC, exon 5 330A-G, exon 11 1241delAC, exon 18 5263G-A, exon 23 5537delA, and exon 24 5625G-T. The mutations in BRCA2 were: exon 11 3374delA, 6076del4bp, 6857delAA, and exon 239254 del5bp. At diagnosis the mean age was 37 years (28-53). Only 3/17 patients presented with involvement of axillary nodes; no patient was diagnosed with metastatic disease. All cases presented infiltrating ductal carcinoma; two of them were medullar carcinoma. Histological grade was available in $13 / 17$ cases, with grade III being the most frequent (12/13). Hormonal receptors were negative in $8 / 10$ patients. The mean follow-up is 129 months (24-224). There were three local recurrences at 17, 108 and 151 months; and two distant relapses at 15 months (complete remission) and at 92 months. Three contralateral BC were diagnosed. HBC has malignant pathological features, but the clinical behaviour seems not to be more aggressive than the sporadic BC in the same age group.

PI-03 A novel BRCA2 gene mutation, 4081 insA, in a Finnish breast/ovarian cancer family associated with favourable clinical course

\footnotetext{
J Hartikainen*, V Kataja† and A Mannermaa*

${ }^{*}$ Chromosome and DNA Laboratory, Bldg 2 C, PO Box 1777, FIN-70211 Kuopio, ${ }^{\dagger}$ Department of Oncology, Kuopio University Hospital, Finland
}

Annually more than 3000 new breast and 400 ovarian cancer cases are diagnosed in Finland. It is estimated that inherited predisposing genes will cause 150-320 cases of breast and 20-40 cases of ovarian cancer each year. In 
Finland 18 different $B R C A 1 / 2$ mutations have been found. Eleven of these mutations are recurrent (including four founder mutations unique to Finland) and one of 18 is unique to one family (4081insA). We have studied 38 Eastern Finnish breast/ovarian cancer families for BRCA1 and BRCA2 germline mutations. The 4081 ins A mutation was detected in PTT analysis and it was confirmed by heteroduplex, sequencing and haplotype analysis. The insertion of an adenine causes frameshift, which leads to a translation terminating signal and protein truncation at codon 1288. The 4081insA mutation is in the BRCA2 gene ovarian cancer cluster region (OCCR) in exon 11. We screened a family of the index patient, her three brothers and four sisters. Mutation was found in three sisters and one brother, in addition to the index patient and one of her three sons. The 4081 insA mutation is rare and has a high penetrance; four of six mutation carriers in the family are affected with breast (two cases) or ovarian cancer (two cases). There were no other cancer cases in the family. Two mutation carriers, both males, at the age of 76 and 59 years, are so far apparently healthy with no symptoms. The four sisters each had an advanced type of breast or ovarian cancer with a poor prognosis. However, they all had a good response to treatment and after 9 to 19 years followup time no recurrence has been seen and all are alive and well. Although the mutation is highly penetrant, the breast and ovarian cancer patients carrying it, in this family, seem to have an exceptionally good clinical course.

PI-04 Breast cancer in young women: prevalence of LOH at p53, BRCA1 and BRCA2

SM Johnson, JA Shaw and RA Walker

Breast Cancer Research Unit, University of Leicester, Clinical Sciences, Glenfield Hospital, Groby Road, Leicester, LE3 9QP

Previous studies have shown that breast cancers show more aggressive pathological features in younger women than those occurring in older women. These findings have raised the question whether differences are present at the molecular level. In order to examine genetic alterations associated with early onset breast cancer 31 cases, selected for age under 35 at diagnosis, were examined for loss of heterozygosity ( $\mathrm{LOH}$ ) and microsatellite instability (MI) in three key chromosomal intervals: 17p13 (p53), 17q11-22 (BRCA1) and 13q12-14 (BRCA2). The cases selected had no obvious family history. DNA was extracted from formalin-fixed paraffin embedded normal and tumour tissue (whole section and microdissected DNA) and analysed by PCR amplification of microsatellite repeat markers. Products were resolved on $10 \%$ nondenaturing polyacrylamide gels and silver stained.

28/31 (90\%) cases exhibited LOH for at least one marker and $19(61 \%)$ cases showed LOH at 2 or more markers. There was no Ml detected. The frequency of $\mathrm{LOH}$ detected for each of the markers was as follows: 17p, D17S796 (37\%) and D17S799 (61\%); 17q, D17S855 (65\%) and THRA1 (41\%); and 13q, D13S171 (48\%). These frequencies are higher (apart from THRA1) than those previously reported for unselected series of breast cancer. Other markers are currently being investigated. These results suggest that $\mathrm{LOH}$ at these regions could be related to early onset breast cancer and to poor tumour prognosis.

\section{PI-05 BRCA1 mutation analysis in breast and ovarian cancer families from Greece}

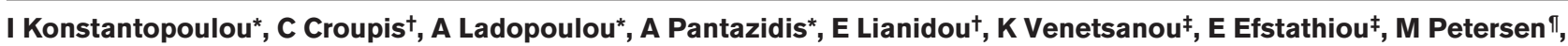
J Janinis ${ }^{\ddagger+\dagger}$, G Fountzilas ${ }^{\S+\dagger}$, E Chiotellis* and D Yannoukakos*

*Molecular Diagnostics Lab, I/R-RP, National Center for Scientific Research 'Demokritos' Athens, 'Laboratory of Analytical Chemistry, Department of Chemistry, University of Athens, ${ }^{\ddagger}$ Agii Anargyri Cancer Hospital, Athens, "Department of Genetics, Institute of Child Health Athens, §AHEPA Hospital, Aristotle University of Thessaloniki Medical School, ${ }^{+\dagger}$ Hellenic Cooperative Oncology Group, Athens, Greece

We have been constructing a genomic DNA database from breast/ovarian cancer patients with a family history, in collaboration with several Greek hospitals. The criteria used for the selection of high-risk families are those accepted universally. In the present study we report three frameshift mutations in BRCA1. These mutations were found in female patients with a family history of breast/ovarian cancer, and are all located in exon 11. Mutation identification was made using PTT and direct sequencing. The first mutation identified is 3741 ins $A$ (codon 1208), carried by a woman who developed bilateral breast cancer at age 31 with her mother's sister affected with breast cancer at age 35 . This mutation is reported only once in the BIC database. The second case is a woman with ovarian cancer carrying the mutation 1623del5-TTAAA (codon 502). Her daughter developed breast cancer and her mother endometrial cancer. This mutation has been reported 6 times in the BIC database. The third BRCA1 mutation, 3099delT (codon 994) is a novel mutation and was found in a woman with ovarian cancer at age 33, with her sister and mother affected with ovarian cancer at different ages. Her grandmother was also affected with breast and ovarian cancer. We have also screened breast/ovarian patients with a family history for two mutations with strong founder effects, 22 patients for 185 delAG and 26 patients for 5382insC. None of these mutations was found, indicating that their frequency in Greece might be quite different from those reported by 
Olah et al [1] concerning Central and Eastern Europe. Mutation analysis of more breast/ovarian patients is in progress. This is the first report of $B R C A 1$ deleterious mutations identified in Greece.
Reference

1. E. Olah et al: Abstract book, A22. BCLC meeting, Nov 28-Dec 1, 1999.

PI-06 BRCA1, BRCA2 and pedigree genetic analysis to determine genetic risk in the UK Royal Marsden Hospital tamoxifen prevention trial

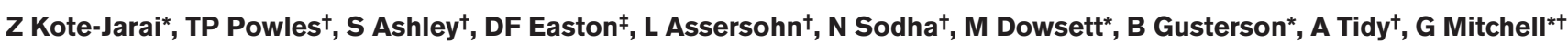
and RA Eeles*+

*Institute of Cancer Research, Sutton, Surrey, SM2 5NG, ${ }^{\dagger}$ Royal Marsden NHS Trust, Sutton, Surrey, SM2 5PT, ${ }^{\ddagger}$ CRC Genetic

Epidemiology Unit, Strangeways Research Laboratories, Cambridge, CN1 4RN, UK

In the Royal Marsden Hospital tamoxifen prevention study, 2500 women at increased risk of developing breast cancer because of family history of the disease were randomised to receive tamoxifen $20 \mathrm{mg}$ daily or placebo for 8 years. 70 woman developed primary breast cancer; 36 whilst on placebo, 34 on tamoxifen. Family history out to at least 2 nd degree relatives was taken from all women in the study. DNA from peripheral blood from 67 of the 70 women was analysed for coding mutations in the BRCA1 and $B R C A 2$ genes by CSGE analysis of the entire coding region of both genes. 7 mutations were found, 2 in $B R C A 1$ and 5 in BRCA2; 4 would be expected to be pathogenic as these were nonsense/frameshifts. 3 were rare variants which were not present in 100 normal controls. The posterior probability of carrying a breast cancer predisposition gene in the individuals who developed breast cancer was assessed using the Cyrillic genetic risk package, based on the Claus model. 26 women had $<50 \%$ posterior probability of harbouring a breast cancer predisposition gene and 44 had a $\geq 50 \%$ chance of having a breast cancer predisposition gene. In the former group of 26 women, 8 had been taking tamoxifen and 18 placebo. In the group of women with $\geq 50 \%$ probability of having a breast cancer gene, 26 had been taking tamoxifen and 18 placebo. The differences between the numbers of women taking tamoxifen who subsequently developed cancer in the two groups divided by $<50 \%$ or $\geq 50 \%$ genetic risk was significant $(P=0.04)$. These preliminary data suggest that tamoxifen prevention may be more effective in women with a $<50 \%$ chance of harbouring a breast cancer predisposition gene. A meta analysis of the interaction of genetic status with tamoxifen chemoprevention effectiveness should be conducted to test this hypothesis.

PI-07 Mutational analysis of BRCA1 and BRCA2 genes in Spanish women with early-onset breast cancer

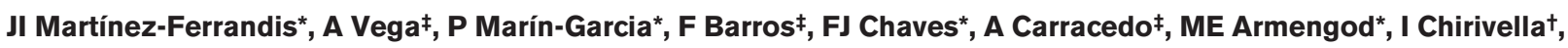
A Lluch ${ }^{\dagger}, \mathrm{J}$ García-Conde ${ }^{\dagger}$ and A Cervantes ${ }^{\dagger}$ *Instituto de Investigaciones Citológicas, 46010 FVIB, Valencia, ${ }^{+}$Hospital Clínico Universitario, 46010 Valencia, ${ }^{\ddagger}$ Unidad de Medicina Molecular, Hospital de Conxo, Santiago de Compostela, Spain

Germ line mutations in the $B R C A 1$ and $B R C A 2$ genes predispose women to breast cancer. The prevalence of $B R C A 1$ and BRCA2 mutations in patients with breast cancer who were unselected for a family history has not been determined in the Mediterranean area. We have screened for BRCA1 and BRCA2 mutations 110 women diagnosed with breast cancer before age 40 years in order to determine the prevalence of these mutations. This screening was performed by using PCR-SSCP analysis of multiplexes and DNA fragments resulting from digestion of about 1300 bp-long PCR products with restriction endonucleases. Sequencing of abnormal bands was used to identify mutations.
Mutations that are predicted to encode truncated protein were detected in $7(6.3 \%)$ of 110 women with early onset breast cancer (2 BRCA1 and 5 BRCA2). Mutations that encode missense amino acid change were detected in 8 $(7.2 \%)$ (4 BRCA1 and 4 BRCA2). This information is important because it determines the cost-benefit implications of genetic testing. We predict that BRCA1 and $B R C A 2$ mutations are approximately equal in our population. A low proportion of the early-onset breast cancer is attributable to mutations in these genes. Clinical and histological features of these women carrying BRCA 1 BRCA2 mutations will be presented. 


\title{
PI-08 One test to identify $50 \%$ of all Norwegian BRCA1 mutations
}

P Møller*, E Hovig*, K Lycke*, RAP Stacy ${ }^{\dagger}$, OJ Dahlberg ${ }^{\dagger}$, A Hagen $^{\ddagger}$, B Hagen, K Løvslett ${ }^{\ddagger}$, H Qvist* and K Heimdal*

${ }^{*}$ The Norwegian Radium Hospital, N-0310 Oslo, ' GenoVision AS, Gjerdrumsvei 12a, 0486 Oslo, ${ }^{\ddagger}$ Regional University Hospital, N-7600 Trondheim, "Central Hospital, N-4000 Stavanger, Norway

In Norway, 1675delA, 1135insA and 816delGT together account for more than half of all BRCA1 mutations in breast-ovarian cancer kindreds (Disease Markers 1999, 15:79-84). We have set up a system for determining absence/presence of these three mutations. DNA is isolated from frozen, whole blood using the GenoPrep instrument for automated nucleic acid purification. The automated system is based on the use of magnetic microparticles, giving high quality DNA for PCR-reactions. The instrument can process 48 samples at a time. The system is designed to handle $100 \mu \mathrm{l}$ blood per sample and produces quantities of DNA sufficient for at least 100 PCR reactions (approximately $3 \mu \mathrm{g}$ ). A multiplex PCRbased fragment analysis has been established employing 30 PCR cycles on a GeneAmp System 7600 cycler (PE-AB). The resulting three fragments are subjected to gel electrophoresis after denaturation by means of the Alf Express $^{\text {TM }}$ (Pharmacia Biotech) for $160 \mathrm{~min}$, and all fragments scored for size variations due to insertions/deletions. The normal product sizes for the three amplified fragments are 85 bp ( $816 \mathrm{del}$ ), $140 \mathrm{bp}$ (1135insA) and $63 \mathrm{bp}$ (1675delA), respectively. Probable mutants are verified by sequencing.

Norwegian health authorities have confirmed that the treating physician may obtain informed consent and obtain blood for mutation analysis; the patient is referred to genetic counselling when a mutation is demonstrated. The activity will define patients and families with mutations for health care, it is cost effective (Disease Markers 1999, 15:167-173), and we may obtain population-based estimates of prevalences, penetrances and expressions of the mutations.

\section{PI-09 Germline TP53 mutations in Finnish breast cancer patients}

\author{
K Rapakko*, M Allinen*, K Syrjäkoski†, P Vahteristo‡, P Huusko*, K Vähäkangasđ, H Eerolaş, T Kainu†, O-P Kallioniemi†, \\ H Nevanlinna ${ }^{*}$ and R Winqvist* \\ Departments of ${ }^{*}$ Clinical Genetics, and ПPharmacology and Toxicology, University of Oulu/Oulu University Hospital, Oulu, \\ +Laboratory of Cancer Genetics, Institute of Medical Technology, Tampere University Hospital, Tampere, Departments of \\ ¥Obstetrics and Gynecology, and §Oncology, Helsinki University Central Hospital, Helsinki, Finland
}

Breast cancer is the most frequent malignancy among women, positive family history being one of the strongest risk factors. Mutations in BRCA1 and BRCA2 account for a portion of inherited predisposition to breast cancer, but recently it was discovered that mutations in these two genes were less common in the studied Finnish cancer families than expected. Therefore, mutations in other susceptibility genes have actively been searched for. TP53 is a tumor suppressor gene that is often found mutated in the Li-Fraumeni syndrome (LFS). Germline alterations of TP53 are also thought to cause predisposition to breast cancer. In a previous study by Huusko et al [1], we screened Finnish LFS families for TP53 exon 5-8 mutations and detected two, Tyr220Cys (exon 6) and Asn235Ser (exon 7), both of which appeared to be associated with accumulation of female breast cancer cases in particular.

We have now screened 130 BRCA1 and BRCA2 negative breast cancer patients from 104 families for germline TP53 mutations covering the whole protein-encoding region of the gene. Our criteria for inclusion were: three or more cases of breast cancer in first- or second-degree relatives, early onset of the disease ( $<35$ years), bilateral breast cancer, or multiple tumors including breast cancer in the same individual. The screen for mutations was performed using conformation sensitive gel electrophoresis
(CSGE) or fluorescence-CSGE (F-CSGE). One missense mutation (Arg248Gln in exon 7) was identified in a family with multiple cases of breast cancer, one of which was bilateral, at very young age of onset. All three Finnish TP53 germline mutations have previously been observed in LFS and breast cancer patients with bilateral tumors or young age of onset. To characterize further the nature of the identified mutations, control DNAs from 500 unselected consecutive breast cancer cases were studied. No additional mutations were found, supporting the notion that these TP53 germline alterations are disease-related. These mutations were also searched for in additional breast cancer families originating from the same geographical regions as those with mutations, but there was no evidence of founder effects that otherwise are common in hereditary diseases in Finland. Our results indicate that the breast cancer-related germline TP53 mutations mainly occur at specific mutation-prone regions of conserved regions of the gene (exons 6-7), and explain a small additional fraction of the BRCA1 and BRCA2-negative breast cancer cases.

K Rapakko and M Allinen made an equal contribution to this work.

\author{
Reference \\ 1. Huusko P et al: Cancer Genet Cytogenet 1999l, 112:9-14.
}




\section{PI-10 X chromosome inactivation pattern in female patients with breast cancer}

\section{Kristiansen*, A Langerød ${ }^{\dagger}$, GP Knudsen*, BL Weber ${ }^{\ddagger}$, A-L Børresen-Dale ${ }^{\dagger}$ and KH Ørstavik*}

${ }^{*}$ Department of Medical Genetics, Ullevål University Hospital, Oslo, ${ }^{\dagger}$ Department of Genetics, Norwegian Radium Hospital, Oslo, Norway, ${ }^{\ddagger}$ Cancer Centre, University of Pennsylvania, Philadelphia, USA

In females one of the two $X$ chromosomes is inactivated in early embryonic life, thus making females mosaics for two cell lines. Most females have a 50:50 distribution of the two cell lines. A deviation from this distribution is called a skewed $X$ inactivation. Skewed $X$ inactivation may be a result of a chance event, due to genetic factors or a selection mechanism. Older females have an increased frequency of skewed $\mathrm{X}$ inactivation in peripheral blood cells. An association between skewed $X$ inactivation and $B R C A 1$ mutation in germline DNA of patients with ovarian cancer was recently reported (Buller et al 1999). A significant increase in skewed $\mathrm{X}$ inactivation pattern was also found in patients with invasive cancer compared to patients with borderline cancer and healthy controls, indicating that skewed $\mathrm{X}$ inactivation is a predisposing factor for the development of invasive ovarian cancer.
We have analysed $X$ inactivation pattern in peripheral blood from 216 female patients with breast cancer and 26 cancer patients with documented BRCA1 germline mutation. Controls were female blood donors. X-inactivation was classified as skewed when $90 \%$ or more of the peripheral blood cells preferentially used one X-chromosome.

Among females with documented $B R C A 1$ germline mutation, $15 \%$ had a skewed $X$ inactivation compared to $8 \%$ of female patients without BRCA1 mutation $(P=0.20)$ and $1 \%$ of control females $(P=0.008)$. Females who developed breast cancer at young ages (25-45 years) had a significantly higher frequency of skewed $\mathrm{X}$ inactivation than control females of the same age group $(P=0.009)$. A germline mutation in an $\mathrm{X}$ chromosome tumour suppressor gene could give a proliferative advantage to cells with this mutation on the active $\mathrm{X}$ chromosome, thus causing skewed $\mathrm{X}$ inactivation.

\section{PI-11 HRAS1 minisatellite alleles and breast cancer in Spanish women under age forty years}

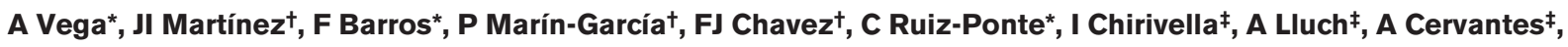
I García-Conde $\ddagger$, ME Armengod ${ }^{\dagger}$ and A Carracedo*

*Unidad de Medicina Molecular, Hospital de Conxo, Santiago de Compostela, 'Instituto de Investigaciones Citológicas 46010, FVIB, Valencia, ${ }^{\ddagger}$ Hospital Clínico Universitario, 46010, Valencia, Spain

Some rare genetic variants in a variable tandemly repeated region (minisatellite) of the $\mathrm{H}$-ras gene have been associated with increased risk of cancers, including breast cancer. The aim of this work is to examine the possibility that rare alleles of HRAS1 minisatellite are implicated in the predisposition to develop early-onset breast cancer.

Methods: One hundred and nine blood samples of a control population from healthy donors, and 95 samples from unrelated women under 40 years old at diagnosis of a first primary breast cancer, have been studied for HRAS1 minisatellite locus. The analysis of HRAS1 alleles was performed using fluorescent detection of size alleles and MVR-PCR.

Results: After the analysis of the HRAS1 MVR sequences and the length polymorphism typing in the healthy control population and the affected patients, we have observed that $20 \%$ of breast cancer patients had at least one rare HRAS1 allele compared to $6.42 \%$ of HRAS1 alleles in the control population $\left(\chi^{2}, P=0.0037\right)$. Therefore, the risk of developing breast cancer increases with the presence of rare alleles $(\mathrm{OR}=3.64$ and $95 \% \mathrm{Cl}=1.46-9.09)$. Only $11.58 \%$ of breast cancer patients studied showed HRAS1 intermediate alleles, an important decrease compared with $25.69 \%$ of intermediate alleles found in the control population.

Conclusions: Our results suggest that the frequency of rare HRAS1 alleles is increased in early-onset breast cancer women, in comparison with a control population. There is also an important decrease in intermediate alleles in the breast cancer population.

\section{PI-12 Germline BRCA2 and somatic P53 alterations in male breast cancer}

\section{B Csokay, N Udvarhelyi and E Olah}

National Institute of Oncology, Department of Molecular Biology, Budapest, Hungary

Cancer of the male breast is infrequent, accounting for less than $1 \%$ of all breast cancer cases in the Western world. Hungary is leading in the mortality rate for this disease in continental Europe. In our recent report [1] a high frequency (33\%) of germline BRCA2 mutations was detected among Hungarian male breast cancer cases without family history. In the current study our aim was to extend these preliminary data on BRCA2 germline alter- 
ations and determine additional somatic genetic changes in both BRCA2 carrier and non-carrier male breast cancers. P53 expression was studied in samples of 32 male breast cancer patients by immunohistochemical analysis using DO-7 and BP-53 antibodies. Unexpectedly, no sample showed overexpression of the P53 protein by either of the antibodies used in our series. To determine whether lack of overexpression was due to absence of mutations in p53, we carried out mutation analysis of the gene using SSCP and direct sequencing of the variants. Updated results of this analysis will be presented.

\section{Acknowledgement}

This work was supported by Hungarian Research Grants ETT (T01008/99) to BC and OTKA (T030039) and ETT (256/1996) to EO.

Reference

1. Csokay et al: Cancer Res 1999, 59:995-998.

\section{PI-13 Constitutional alterations of 9p23-24 in independent BRCA2 breast cancer families}

\section{Schwab, L Savelyeva, A Claas, H An and S Brouwers}

Division of Cytogenetics, German Cancer Research Center, 60120 Heidelberg, Germany

Germ line mutations of $B R C A 2$ are predicted to account for the majority of families with both male and female breast cancer. However, there is circumstantial evidence that the cancer risk conferred by BRCA2 mutation may be modified by other genetic or environmental factors. By employing a combination of classical G-banding and fluorescence in situ hybridization analyses we have identified chromosomal alterations on 9p23-24 in peripheral lymphocytes of independent BRCA2 breast cancer patients. Tandem duplication and amplification with inversion are constitutional rearrangements in four male breast cancer patients from two high-risk families. Interstitial deletion of the same region was found in four male and one female patients from an independent family. The biological significance of the coexistence of BRCA2 mutation and 9p23-24 abnormalities in breast cancer families may be complex. Possible explanations include (1) the BRCA2 mutation is related to the 9prearrangement, or (2) the $9 p$ rearrangement is elicited by another as yet unknown factor, and chromosomal changes on $9 p$ could be related to modifying cancer risk.

PI-14 kConFab: a unique Australasian research initiative for familial breast cancer

\section{J Sambrook}

Peter MacCallum Cancer Institute, Melbourne, Australia

The Kathleen Cuningham Foundation Consortium for Research into Familial Aspects of Breast Cancer (kConFab) is a unique Australasian research co-operative which brings together geneticists, clinicians, surgeons, scientists, pathologists, psychologists, oncologists and epidemiologists from 32 institutions in New Zealand and the five mainland States of Australia. The aims of the Consortium are to identify Australasian families with predisposition to breast/ovarian cancer through Familial Cancer Clinics in Australia and New Zealand, to identify the predisposing genes and characterise germline mutations. The study aims to determine the effect of the type of mutation and the influence of lifestyle and environmental factors on the type, age of onset and severity of cancer, the response of the patients to treatment and the psychosocial consequences. In addition to genetic, epidemiological and medical data, kConFab obtains and stores clinical samples, DNA, RNA, tumour and prophylactically removed tissue. All data are stored in a relational database that is accessible for approved basic and clinical research projects.
As of January 2000, kConFab has identified over 700 Australasian families, who have presented at Family Cancer Clinics with exceptionally severe histories of breast or breast/ovarian cancer and features suggesting a dominantly-inherited predisposition to the disease. To date, over 2200 individuals have consented to donate blood and complete questionnaires concerning their health, diet and lifestyle. By the end of the accrual phase of the study (2002), kConFab expects to have accumulated genetic and epidemiological information on at least 7000 members of high-risk families, including first and second degree relatives of all mutation carriers and individuals affected with breast or ovarian cancer. Further information about $k$ ConFab may be found on our website at http://www.pmci.unimelb.edu.au/kconfab.

Acknowledgement: On behalf of the Kathleen Cuningham Foundation Consortium for Research into Familial Breast Cancer (kConFab).

\section{PI-15 Germline mutations of BRCA1-associated RING domain (BARD1) gene in breast and/or ovarian} families negative for BRCA1 and 2 alterations

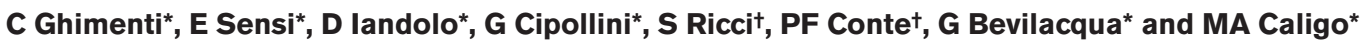

Department of Oncology, *Division of Pathology and +Division of Medical Oncology, University of Pisa, Italy

BARD1 (BRCA1-associated RING domain) has been identified by yeast two-hybrid screening as a protein specifically interacting with the product of BRCA1 gene. Somatic and germline mutations of BARD1 have been 
detected in sporadic breast, ovarian and endometrial cancers. In this study, we evaluate the frequency of BARD1 germline mutations in 20 Italian hereditary breast and breast-ovarian families tested negative for BRCA1 and BRCA2 mutations. Two families were breast-ovarian, $11 \mathrm{had}$ more than four cases of breast cancer and five had only two affected in the family. Mutational analysis was performed by SSCP for the entire coding region and exonintron splice boundaries of BARD1 gene. Direct sequence analysis was used to identify the genetic alterations. We found three different germline alterations of the BARD1 gene, two missense and one frameshift: a $G>C$ transversion in codon 557 that produces an aminoacidic change Cys $>$ Ser in exon 7; a $A>G$ transition in codon 295 that produces an aminoacidic change Asn $>$ Ser in exon 4; a $21 \mathrm{bp}$ deletion after nucleotide 1071 that produces an inframe deletion of 7 aminoacid in exon 4.
A group of 20 sporadic breast cancers below 40 years of age, selected as a control group was analyzed. We found only a somatic mutation in one tumor.

The mutation was the same in-frame deletion found in the family group. A study of loss of heterozigosity of BARD1 locus (D2S143, D2S164 and D2S295 markers) in the tumor tissues of patients carrying the BARD1 mutations is under investigation.

These data suggest that BARD1 could be involved in the susceptibility of hereditary breast and ovarian tumors.

This work was supported by AIRC.

\section{PI-16 Mammary-specific targeting of the murine BRCA2 breast cancer susceptibility gene in mice} NK Collins, KA McAllister, LM Bennett, K-U Wagner, T Ward, J Malphurs, G Goulding, EM Eddy, BJ Davis and RW Wiseman National Institute of Environmental Health Sciences, NIH, Research Triangle Park, NC 27709 and NIDDK, NIH, Bethesda, MD 20892, USA

Women with inherited mutations in the BRCA2 gene have a very high lifetime risk of developing breast cancer. Previously, we used gene targeting with embryonic stem cells to generate mice with a mutation that disrupts exons 10 and 11 of the Brca2 gene. Mice that are homozygous for this mutation exhibit an embryonic lethal phenotype. To overcome this difficulty we have generated mice with loxP sites flanking Brca2 exon 27. Prior studies have shown this C-terminal domain of Brca2 interacts with Rad51, and cells that lack Brca2 exon 27 are hypersensitive to gamma-radiation. Therefore, site-specific recombination of loxP sites and deletion of exon 27 in this floxed Brca2 allele by a Cre recombinase should disrupt basic functions of Brca2 in DNA repair. The mammary-gland-specific removal of Brca2 exon 27 by Cre-mediated recombination in vivo has been accomplished by crossing the homozygous floxed Brca2 mice with a mouse mammary tumor virus (MMTV)-Cre strain D transgenic mice. Analyses of
ROSA26 LacZ Cre reporter mice confirm that this MMTVCre transgene is specifically activated at the onset of puberty in mammary epithelial cells. In parallel studies a germline deletion of exon 27 was created by transiently electroporating embryonic stem cells carrying the floxed Brca2 allele with a Cre-expression plasmid. Surprisingly, mice homozygous for the germline deletion of exon 27 appear to be completely viable at birth, but preliminary studies suggest impaired male fertility. Gross phenotypic abnormalities in mammary gland ductal morphogenesis have not been shown by mammary whole mount preparations in these animals at up to six months of age. These mice are being observed closely for neoplastic development in mammary glands as well as other tissues. Mammary-specific Brca2 ${ }^{\Delta 27}$ mice should be a valuable experimental model mimicking the breast tumor development of women who have inherited a BRCA2 defect and then acquire a secondary somatic BRCA2 mutation.

PII-01 Relative expression of progesterone receptors A and B in premalignant and invasive breast lesions

\section{P Mote and C Clarke}

Westmead Institute for Cancer Research, University of Sydney, Westmead, NSW 2145, Australia

Progesterone is critical in mammary gland development. Breast cancer evolves from normal tissue through increasingly abnormal cellular changes that include increased expression of progesterone receptor (PR), and PR is an established marker of response to endocrine therapy. $P R$ is expressed as two proteins (PRA and PRB) with different functions, and in vitro evidence reveals PRA to inhibit PRB function. This suggests that PRA may repress progesterone action and that the ratio of PRA:PRB may be an important determinant in tissue sensitivity to ovarian steroid hormones.
This study examined the expression of PRA and PRB proteins in normal breast tissue $(n=13)$ during the menstrual cycle, and in premalignant $(n=45)$ and malignant $(n=39)$ breast tissues, to determine differences in relative isoform expression. We used dual immunofluorescent histochemistry on formalin-fixed, paraffin-embedded tissue sections using mouse monoclonal antibodies that bind to either PRB or PRA.

In most normal breast cases PR staining was confined to scattered epithelial cells expressing equivalent levels of 
PRA and PRB. However, $50 \%$ of cases in the luteal phase $(n=6)$ showed reduced PRA expression. In proliferative premalignant lesions without atypia (PDWA, $n=15$ ), there was a marked increase in intensity and number of cells expressing PR, but inter-cell homogeneity was maintained. Atypical proliferative benign lesions (ADH, $n=15$; DCIS, $n=15$ ), showed high levels of both PRA and PRB expression with notable inter-cell heterogeneity in relative isoform content. This was also observed in malignant breast tumours $(n=39)$. Furthermore, breast tumours expressing an overall predominance of one isoform were associated with features of higher histological grade.

In conclusion, our results show a change from inter-cell homogeneity of PRA:PRB in normal tissue to extensive heterogeneity in the malignant state, suggesting a progressive loss of control of relative PRA and B expression that may occur early in cancer development and may eventually be associated with features of poorer prognosis.

PII-02 Growth inhibition and growth stimulation by estradiol of estrogen receptor transfected human breast epithelial cell lines involve different pathways

\title{
BK Lundholt, P Briand and AE Lykkesfeldt
}

Department of Tumor Endocrinology, Institute of Cancer Biology, Danish Cancer Society, Strandboulevarden 49, DK-2100

Copenhagen $\varnothing$, Denmark

Epidermal growth factor (EGF) and estradiol (E2) are important mitogens in breast epithelial cells, and expression of epidermal growth factor receptor (EGFR) and estrogen receptor (ER) is often inversely correlated in human breast cancer cells. Stable transfection of ER-negative cells with ER cDNA is not sufficient to restore E2-mediated growth stimulation, suggesting a disturbance of this inverse correlation in ER-transfected cell lines. In this study we used the ER-transfected human breast epithelial cell lines HMT$3522 \mathrm{F9}$, growth inhibited by E2 in the presence of EGF, and HMT-3522F9/S3B, growth stimulated by E2 in the absence of EGF. The E2-mediated growth regulatory differ- ences of the cell lines were not due to altered expression of EGFR, TGF $\alpha$, or c-erbB2 mRNA. A decreased MAP kinase activity was observed in HMT-3522F9 cells in response to $\mathrm{E} 2$, indicating that in these cells altered cross-talk between the ER and the EGFR/MAP kinase signalling pathway could be due to the E2-stimulated growth inhibition. Interestingly, no changes in EGFR, ErbB2 or MAP kinase activity was observed in E2-stimulated in HMT-3522F9/S3B cells in response to E2, suggesting a MAP kinase-independent E2mediated growth stimulatory mechanism. We are currently investigating the pathway(s) involved in the E2-mediated growth stimulation of HMT-3522F9/S3B cells.

PII-03 Hairy and enhancer of split homolog-1 (HES-1) mediates the proliferative effect of 17 $\beta$-estradiol on breast cancer cell lines

\author{
A Ström, N Arai, J Leers and J-Å Gustafsson \\ Karolinska Institutet, Department of Biosciences at Novum, S-141 57 Huddinge, Sweden
}

The mechanism behind estradiol-dependent growth of breast cancer is presently not well understood. We show that the hairy and enhancer of split homolog-1 (HES-1) protein level in the breast cancer cell lines T47D and MCF-7 is down-regulated by $17 \beta$-estradiol treatment. This regulation could be reversed by addition of the anti-estrogens $4 \mathrm{OH}$ tamoxifen, raloxifen and Imperial Chemical Industries (ICl) 182,780. Furthermore, T47D cells with inducible exogenous HES-1 expression showed that HES1 protein needs to be removed in order for $17 \beta$-estradiol to have a proliferative effect and subsequently up-regulating proliferating cell nuclear antigen (PCNA).

An inverse correlation between the protein levels of HES-1 and PCNA was found in colon cancer cell lines. These findings point to a role of HES-1 as a tumor suppressor in epithelial cells, and as a target for 17 $\beta$-estradiol in breast cancer cells. Present findings makes HES-1 useful for diagnosis and an interesting target for cancer treatment.

PII-04 Genetic variants of CYP19 (aromatase) and breast cancer risk

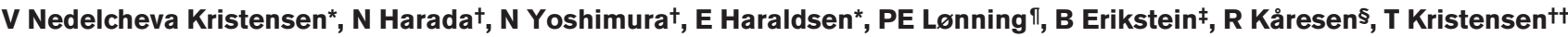 \\ and A-L Børresen-Dale* \\ ${ }^{*}$ Department of Genetics, ${ }^{\ddagger}$ Department of Oncology, Institute of Cancer Research, The Norwegian Radium Hospital, Montebello \\ 0310, Oslo, Norway, ${ }^{\dagger}$ Department of Biochemistry, School of Medicine, Fujita Health University, Toyoake, Japan, "Department of \\ Oncology, Haukeland Hospital, University of Bergen, §Department of Oncology, Ulleval Hospital, Oslo, ${ }^{++}$Department of \\ Biochemistry, University of Oslo, Norway
}

The effect of an SNP in exon 10 of CYP19 on tumour mRNA levels and splice variants was studied and corre- lated with clinical parameters and risk of breast cancer. In the vast majority of breast cancers, the estrogen levels 
modulate the tumour growth and depend on the activity of CYP19. Patients $(n=481)$ and controls $(n=236)$ were genotyped by $\mathrm{T}$-tracks in a single sequencing reaction (SSR). The frequency of TT genotypes was significantly higher in patients versus controls $(P=0.007)$ particularly among those with stage III and IV disease $(P=0.004)$ and with tumours larger than $5 \mathrm{~cm}$ $(P=0.001)$. A significant association between presence of the $\mathrm{T}$ allele and the level of aromatase mRNA in the tumours was observed $(P=0.018)$, as well as with a switch from adipose promoter to ovary promoter $(P=0.004)$. Previously, we reported a rare polymorphic allele of CYP19 (repeat (TTTA) ${ }_{12}$ ) to be significantly more frequent in breast cancer patients than in controls. Here we describe another polymorphism, a C-T substitution in exon 10 of the CYP19 gene which is in strong linkage disequilibrium with the (TTTA) $n$ polymorphism but with higher frequency of the variant allele. Our data suggest that the T-allele of the CYP19 gene is associated with a 'high activity' phenotype.

PII-05 The involvement of the MAPK signalling pathway in the adaptation of MCF-7 cells to long-term oestrogen deprivation

\section{L-A Martin, CMW Chan, C Marshall and M Dowsett}

Institute of Cancer Research, Fulham Road, London, UK

The molecular mechanism associated with the transition of breast tumours to steroid hormone-independent growth is poorly understood. However, a number of studies have demonstrated the potential role of the mitogen-activated protein kinase (MAPK) signalling pathway in the initiation and pathogenesis of breast cancer.

In an attempt to study the transition to oestrogen-independent growth, wild-type (wt) MCF-7 cells were cultured in oestrogen (E2)-deficient medium for over 100 weeks. During this time the cells were characterised and shown to pass through three distinct phases. Quiescent (LTEDQ), followed by an increase in basal growth rate paralleled by hypersensitivity to E2 (LTED-H), and finally transition to an E2-independent phase (LTED-I). Western blot analysis of the LTED cells showed elevated levels of ER $\alpha$ compared to the wt MCF-7 cells. The ER $\alpha$ was phosphorylated on serine 118 despite absence of E2.

Assessment of the level of activated MAPK showed a mean 7-fold increase in LTED-Q, 4- fold in LTED-H and 6- fold in LTED-I compared to the wt MCF-7 cells. As the LTED-I phase progressed MAPK levels fell, but after 90 weeks remained $30 \%$ higher compared to wt MCF-7. Suppression of MAPK activity in LTED-I cells, using a MEK inhibitor, significantly reduced but did not block ER $\alpha$ phosphorylation. Similarly transfection of LTED-I cells with an E2 responsive reporter construct (pEREtkCAT), followed by treatment of the cells with a MEK inhibitor, resulted in a $50 \%$ decrease in basal ER $\alpha$ transcription. However, a combination of E2 and the MEK inhibitor suppressed ER $\alpha$-directed transcription by only $30 \% \mathrm{com}$ pared to E2 alone.

These data support previous findings that elevated MAPK levels are found during ligand-independent cell proliferation. However, this is unlikely to be the sole pathway operating to achieve this adaptation; rather a complex network of kinases and molecular switches may operate at different temporal stages during long-term oestrogen deprivation.

PII-06 Phosphorylation of human progesterone receptors by MAPKs mediates transcriptional synergy between progestins and MEKK1 in human breast cancer cells

CA Lange*, T Shent and KB Horwitz ${ }^{\dagger}$

*University of Minnesota Cancer Center, Minneapolis, MN, and ${ }^{\dagger}$ Division of Endocrinology, University of Colorado Health Sciences Center, Denver, CO, USA

Breast cancers that are steroid-hormone resistant often overexpress growth factor receptor tyrosine kinases, including members of the type I (EGFR/Erb2) family. Cross-talk between growth factor- and progesteronemediated signal transduction pathways may contribute to the development of resistance to steroid hormone-based therapies in breast cancer. To mimic constitutive activation of molecules downstream of growth factor signalling pathways, we overexpressed activated MAP/ERK Kinase Kinase (MEKK) in T47D human breast cancer cells. MEKK is a strong activator of p42/p44, and p38 mitogen activated protein kinases (MAPKs). MEKK expression resulted in 20-fold increased R5020-mediated transcription driven by a co-expressed progesterone-response-element (PRE)containing promoter linked to the luciferase reporter gene; progesterone receptor (PR) levels did not change in the presence of MEKK alone, but decreased in the presence of MEKK and R5020. Potentiation by MEKK of progestininduced transcription also occurred in HeLa cells, and was dependent on the presence of a PRE, and functional PR. PR antagonists RU486 and ZK98299 blocked this effect. The MEK inhibitor, PD98059, also blocked transcriptional synergy between MEKK and progestins, indicating a requirement for p42 and p44 MAPKs. To test 
whether the effect of MAPK activation was due to direct phosphorylation of PR, we expressed MEKK in T47D cells stably expressing either wild-type or mutant $P R$, in which either of two MAPK consensus site serine residues, Ser 294 or Ser 345, were mutated to alanine. Both MAPK mutants of PR were resistant to MEKK- and R5020induced transcriptional synergy, but, like wild-type PR, still responded to progestins alone. Thus, mutant PR are functional in response to progestins, but are incapable of cross-talk with MAPK-driven pathways. Phosphorylation of PR by MAPKs in response to peptide growth factor signalling may increase PR transcriptional activity on PREcontaining growth regulatory genes, thus altering steroidal control of breast cancer cell growth.

Acknowledgement

Supported by NIH grant DK-53825.

\section{PIII-01 The expression of the EGFR family members in breast carcinomas}

\section{Z Suo and JM Nesland}

Department of Pathology, The Norwegian Radium Hospital and Institute for Cancer Research, University of Oslo, Norway

The EGF receptor family members (EGFR, c-erbB-2, cerbB-3 and c-erbB-4) of tyrosine kinase receptors are involved in the regulation of cell growth and differentiation. Differential expression of such members in breast carcinomas may be associated with different clinical behaviours. A series of Norwegian and Chinese breast cancer patients has been examined in our laboratory with the same immunohistochemical protocol. The expression rate of EGFR, c-erbB-2 and c-erbB-3 in these two series was similar, $43.9 \%, 37 \%$ and $22 \%$ in the Norwegian series and $53 \%, 35.8 \%$ and $17 \%$ in the Chinese series, respectively. Comparing the immunohistochemical results in available benign breast material and 'normal' material in breast carcinomas, we conclude that the expression of EGFR, c-erbB-2 and c-erbB-3 is increased in breast malignancy. The expression rate of c-erbB-4 was lower in the Chinese series, $45.7 \%$ vs $81 \%$. That was probably due to the fact that there were more grade III tumours in the Chinese series, since the expression of c-erbB-4 was found to be inversely associated with histological grade in invasive ductal carcinomas. Compared with the expression in benign and normal breast tissues, the expression of $\mathrm{C}^{-}$ erbB-4 was, however, decreased in breast carcinomas in both series. Available follow-up data in the Chinese series demonstrated that c-erbB-4 expression in node-negative breast carcinomas was associated with better prognosis, but not c-erbB-4 expression in node-positive carcinomas. It is concluded that while the expression of EGFR, c-erbB2 and c-erbB-3 is associated with cancer development, the expression of c-erbB-4 may have a different role.

\section{PIII-02 IGFBP-7 expression in human breast cancer and association with proliferation and cell cycle} aberrations

H Östlund*, A Burger†, A Seth‡ and G Landberg*

${ }^{*}$ Department of Medical Biosciences, Pathology, Umeå University, Sweden, ${ }^{\dagger}$ Tumor Biology Center at the University of Freiburg, Germany, ${ }^{\ddagger}$ Department of Laboratory Medicine and Pathobiology, University of Toronto, Women's College Hospital, Toronto, Canada

The complex insulin-like growth factor network of ligands, receptors and binding proteins has been shown to be disturbed in breast cancer, potentially leading to IGF1 receptor activation and uncontrolled tyrosine kinase signalling. In addition to defects in proteins controlling cell cycle checkpoints, this type of aberrations could affect tumor growth and survival, thereby influencing both tumor aggressiveness and potential response to treatments. We have earlier shown that the $\mathrm{T} 1 \mathrm{~A} 12 / \mathrm{mac} 25$ protein, which is identical to the insulin-like binding protein (IGFBP-7), is differentially expressed in breast cancer cells compared with normal cells. The gene product seemed to be lost in the progression from premalignancy to invasive breast cancer and loss of heterozygosity (LOH) of the $4 q 12-13$ region was frequently observed in invasive cancers, suggesting a suppressor-life function for IGFB-7. In 104 inva- sive breast cancers arranged in a tissue-array system, all with known status of cell-cycle aberrations and clinicopathological data, the expression of IGFB-7 was monitored using immunohistochemistry. Cytoplasmic staining of variable intensity was observed in the tumors and $14 \%$ lacked IGFBP-7 staining, 20\% had low staining, 32\% intermediate staining and 34\% strong staining. Low IGFB7 was associated with high cyclin $E$ expression, retinoblastoma protein inactivation, low bcl-2 and poorly differentiated tumors. There was further a significantly impaired prognosis for patients with low IGFB-7 protein tumors. Interestingly, IGFB-7 was strongly and inversely associated with proliferation (Ki-67 \%) in estrogen receptor-negative tumors, suggesting an important cell-cycle regulatory function for IGFBP-7 separate from the interaction with the estrogen receptor pathway. 


\section{PIII-03 Activation of the Her2/neu receptor leads to antiestrogen resistance, but acquired resistance is not caused by altered ErbB expression and activation}

SS Larsen and AE Lykkesfeldt

Department of Tumor Endocrinology, Institute of Cancer Biology, Danish Cancer Society, Strandboulevarden 49, DK-2100

Copenhagen $\varnothing$, Denmark

Development of acquired resistance against antiestrogen treatment is a serious problem in human breast cancer, and knowledge of alterations resulting in resistance is important for selection of further treatment. To mimic the clinical situation we have established a series of MCF-7 human breast cancer cell lines by long term treatment with the antiestrogens tamoxifen, ICl 164,384, and ICl 182,780 (Faslodex $^{\mathrm{TM}}$ ). Common for these cell lines is a decreased expression of the estrogen receptor $\alpha(E R \alpha)$. In human breast cancer, lack of response to endocrine therapy is often associated with decreased expression of the estrogen receptor and increased expression of epidermal growth factor receptor (EGFR) and/or HER-2/neu (ErbB-2). Our antiestrogen-resistant cell lines did not express altered levels of EGFR, HER-2/neu, ErbB-3 and ErbB-4. Estrogen and antiestrogen regulation of HER$2 /$ neu expression was essentially similar in parent and resistant MCF-7 cells. Treatment with antibodies to HER2/neu (Herceptin ${ }^{\text {TM }}$ ) did not affect growth of MCF-7 cells or resistant cells, indicating that in this in vitro model system, acquired antiestrogen resistance does not emerge from activation of the HER-2/neu signalling pathway. However, addition of heregulin 1- $\beta 1$ abolished the inhibitory activity of ICl 182,780 on MCF-7 cells, demonstrating that activation of the HER-2/neu receptor signalling pathway can override the growth inhibitory effect of ICl 182,780. The effect of heregulin $1-\beta 1$ could be abrogated by Herceptin ${ }^{\mathrm{TM}}$.

PIII-04 Expression of Akt kinases related to ErbB2 and results of adjuvant therapy of breast cancer

\section{O Stal, L Akerberg, B Nordenskjold, B Olsson, LE Rutqvist and L Skoog}

Department of Oncology, University Hospital, Linkoping, Oncologic Center and Division of Cytology, Karolinska Hospital, Stockholm, Sweden

It has been suggested in several studies of breast cancer that overexpression of the growth factor receptor erbB2 is associated with less benefit from certain adjuvant treatments. The mechanisms are not fully understood. The erbB2 receptor activates several signal pathways including the phosphatidyl-inositol-3-kinase (PI3-K)/Akt (also known as PKB) pathway, which is implicated in cell survival. This pathway has shown to be a target of the tumor suppressor PTEN.

We have analysed the expression of Akt1 and Akt2 by immunohistochemistry in frozen tumor samples from 280 postmenopausal patients who participated in a randomised trial comparing CMF chemotherapy and postoperative radiotherapy. The patients were simultaneously randomised to tamoxifen or no endocrine treatment. A marked cytoplasmic staining in more than $10 \%$ of the malignant cells for Akt1 was observed in $24 \%$ of the cases. Thirty percent showed staining for Akt2. Among Akt2-positive tumors, Akt1 was more frequently expressed in erbB2-positive compared with erbB2-negative cases, $62 \%$ and 30\%, respectively $(P=0.005)$. Akt1+/ER+ patients seemed not to benefit from adjuvant tamoxifen (RR $=0.93,95 \% \mathrm{Cl} 0.35-2.8)$, whereas the Akt1-/ER+ group showed a significantly improved distant recurrence-free survival with tamoxifen $(R R=0.51$, $95 \% \mathrm{Cl} 0.31-0.84, P=0.0056)$. No interactions were observed between Akt1 or Akt2 and the result of CMF vs radiotherapy treatment considering distant recurrence-free survival. For locoregional recurrence a high expression of either Akt1 or erbB2 or both significantly predicted a poor benefit from radiotherapy vs CMF $(P=0.038)$.

\section{PIII-05 Anti-HER2 mAb plus calicheamicin immunoconjugate linked on liposomal vinorelbine induces} ADCC and apoptosis in HER2-positive high grade DCIS

J Giannos and P Ginopulos

Department of Clinical Oncology, Regional Hospital, St. Andreas, Greece

Overexpression of the HER2 proto-oncogene which encodes a $185 \mathrm{kDa}$ protein frequently coincides with aggressive and chemoresistant DCIS due to inhibition of PCD after chemotherapy. Also, it is associated with aneuploidy, p53 abnormalities, enhanced DNA repair and synthesis, cell growth, mitotic rate and tumorigenicity. Tissue from high-grade DCIS was excised from a patient, and tumour cells were isolated by the collagenase method. Analysis with IHC showed HER2 overexpression. We prepared immunoconjugates of anti-HER2 mAbs and calicheamicin, which is an apoptotic antibiotic with up to 1000-fold greater potency than the clinically most used anticancer drugs. This immunoconjugate was linked onto pegylated DRV liposomes which contained vinorelbine. After treatment, we observed HER2 downregulation by IHC. TEM exhibited disruption of the microtubular cytoskeleton due to vinorelbine, and $\mathrm{mAb}(\mathrm{Fc})$-directed killing of tumour cells by immune effector cells such as 
macrophages, neutrophils and lymphoid cells such as $\mathrm{K}$ and NK cells, indicating antibody-dependent cellular toxicity (ADCC). Biochemical assays such as MTT exhibited reduced metabolic activity, while BrdU showed great reduction of DNA synthesis. Anti-ssDNA mAbs and binding of Annexine-V with phosphatidyl-serine IHC confirmed that apoptosis was the mechanism of growth inhibition. SEM and TEM showed violent membrane blebbing of apoptotic cells (zeiosis), and fragmentation of DNA creating a vacuolar nucleus due to calicheamicin-damaging action after binding to the third end of oligopurine tracts causing strand breaks. Subsequently, tumour cells break up into apoptotic bodies that adjacent cells recognise and phagocytose due to PS externalisation, indicating a bystander killing effect (BKE). We conclude that by delivering intracellularly cytotoxic agents such as calicheamicin through conjugating to antiHER2 mAbs linked on liposomal vinorelbine, we can circumvent chemoresistance, induce ADCC, inhibit mitosis at metaphase and destroy tumoral DNA, leading to irreversible D2 apoptotic signs with subsequent bystander killing of high grade DCIS.

\section{PIII-06 Factors modulating integrin $\beta$ I expression on the breast cancer cell lines MCF-7 and MDA-MB-} 231

CJ Pogson, CMW Chan, L-A Martin, GPH Gui and M Dowsett

Departments of Academic Surgery and Biochemistry, The Royal Marsden and Institute of Cancer Research, Fulham Road, London, England

The metastatic cascade describes a sequence of cellular events that forms the pathological basis of tumour progression. The integrins are cell adhesion molecules that play a leading role in this complex process. The objective of this study is to improve our understanding of the role of integrins in breast cancer metastasis by investigating how growth factors (Epidermal Growth Factor (EGF) and Insulin-like Growth Factor 1 (IGF-1)), oestradiol (E2) and tamoxifen affect integrin $\beta \mathrm{I}$ expression. The breast cancer cell lines MCF-7 and MDA-MB-231 were used and integrin expression measured by Western Blotting.

We have demonstrated that EGF and IGF-1 up-regulate integrin $\beta 1$ expression on the MDA-MB-231 cell line by 2.7- and 2.8-fold respectively. By doing so these cells may generally become more adhesive and therefore less prone to metastasise. Tamoxifen down-regulated integrin $\beta \mathrm{I}$ expression on this oestrogen receptor (ER)-negative cell line, with maximal effect at $10^{-6} \mathrm{M}$ (34\% reduction). This suggests signalling through an alternative pathway. Integrin down-regulation may render cells less adhesive and therefore less invasive.

High concentrations of E2 significantly up-regulated integrin $\beta \mathrm{I}$ expression on the MCF-7 cell line, whereas low concentrations resulted in a down-regulation, with maximal effects at $10^{-7} \mathrm{M}$ (1.7-fold induction) and $10^{-11} \mathrm{M}$ (29\% reduction) respectively. Conversely, high concentrations of tamoxifen down-regulated integrin $\beta \mathrm{l}$ expression and low concentrations up-regulated expression, with maximal effects at $10^{-8}$ $\mathrm{M}$ (49\% reduction) and $10^{-9} \mathrm{M}$ (2.7-fold induction) respectively. These data provide a cellular basis for the modulation of integrin expression and may explain why some ER-negative patients respond well to tamoxifen. Identification of factors that regulate integrin expression may lead to the development of novel anti-metastatic agents.

\section{PIII-07 HER-2 activation and TP53 inactivation as predictors of survival in breast cancer}

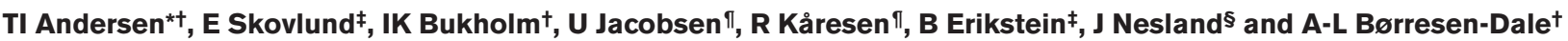 \\ Departments of *Oncology and ^Surgery, Ullevål University Hospital, N-0407 Oslo, Norway. Departments of ${ }^{+}$Genetics, IOncology, and §Pathology, The Norwegian Radium Hospital, N-0310 Oslo, Norway}

Activation of the HER-2 proto-oncogene and inactivation of the TP53 tumour-suppressor gene belong to the most common genetic changes in human breast carcinomas. Both appear to be of prognostic significance, at least in patients with node-positive disease. The relevance of these changes in node-negative disease, however, still remains uncertain. Moreover, the relationship between HER-2 and TP53 status remains to be fully clarified.

HER-2 and TP53 status were determined in 261 breast carcinomas collected from Norwegian breast cancer patients diagnosed between 1984 and 1994. HER-2 status was determined using immunohistochemistry. A subset of the tumours was also examined with regard to gene amplification using the Southern blot technique. A significant association was found between the presence of gene amplification and positive HER-2 immunostaining in the tumour. The tumours were also examined for TP53 alterations using CDGE (constant denaturant gel electrophoresis) and immunohistochemistry. A significant association was found between the presence of HER-2 activation and TP53 inactivation in the tumour. Survival analyses will be presented. 


\section{PIII-08 Herceptin ${ }^{\mathrm{TM}}$ (trastuzumab) in patients who overexpress Her-2 and have failed multiple} chemotherapy regimens for advanced breast cancer: a pragmatic study

\section{G Westman}

The Norwegian Radium Hospital, Montebello, N-0310 Oslo, Norway

This prospective study will include patients who are at least 18 years of age, with a histologically or cytologically confirmed diagnosis of advanced breast cancer (ABC), who have failed or are resistant to treatment with at least two chemotherapy regimens for $A B C$, and who overexpress HER-2. A maximum of 62 patients are to be included for the detection of an overall response rate of 20\% (10-30\%, 95\% confidence interval).

Her-2 status will be analyzed with immunohistochemistry (Novocastra NCL CB-11, mouse monoclonal antibody).
Treatment will be single dose trastuzumab: $4 \mathrm{mg} / \mathrm{kg}$ first treatment, and $2 \mathrm{mg} / \mathrm{kg}$ thereafter, for at least 10 cycles. Tumor response and side effects will be documented. In a separate protocol bone marrow micrometastasis will be analyzed.

The study started at the end of December 1999. Four patients have been included so far. More data and some results will be presented at the meeting.

\section{PIV-01 Role of the breast cancer susceptibility gene BRCA1 in radio-resistance}

\section{Sylvain and Y-J Bignon}

Laboratoire d'Oncologie Moléculaire EA 2145, Centre Jean Perrin, BP392 58 Rue Montalembert 63011 Clermont-Ferrand

Cedex1, France

Evidence that $B R C A 1$ is involved in DNA repair stems from its interaction with proteins like RAD51, RAD50, etc We hypothesised that overexpression or antisense inhibition of the mouse homologue Brca1 in an ovarian cancer cell line could result in changes of sensitivity to $\gamma$ radiation.

Mouse ovarian cancer cell line ID-8 was transfected or infected with Neo-resistant vector or retrovirus expressing full-length Brca1 in the sense or antisense orientation. G418-resistant colonies were isolated (two sense, S2 and S13, and three antisense, AS1, AS2 and AS3) and levels of Brca1 were quantified by real time quantitative RT-PCR. Cells were incubated in 96 -well culture plates at $37^{\circ} \mathrm{C}$ for 24 hours. They were irradiated $(0,1,3,5$ and $10 \mathrm{~Gy})$, incubated at $37^{\circ} \mathrm{C}$ for 24 hours, washed, and postincubated at $37^{\circ} \mathrm{C}$ in fresh medium for 48 hours, after which cell density was measured using a colorimetric assay with sulforhodamine B.
Colonies S2 and S13 respectively expressed very low and high levels of Brca1 transgene, and expression of transgene in colony AS2 was 12- and 60-times higher than in colonies AS1 and AS3 respectively. Overexpression of Brca1 resulted in enhanced resistance to irradiation (1.2 to 1.5-fold), whereas inhibition of expression of Brca1 resulted in a very slight increase in sensitivity. Increased radio-resistance induced by overexpression of Brca1 is consistent with other studies, and supports a role for BRCA1 in DNA damage repair. On the other hand, the discrepancy between our results obtained with inhibition expression of Brca1 and data in the literature (ionising radiation hypersensitivity in human cancer cells containing mutated BRCA1 and mouse embryonic stem cells carrying a Brca1 null mutation) is unexpected. We need to investigate further the inhibition of expression of protein Brca1 and above all its functional properties, analysing expression profiles of $B R C A 1$ downstream target genes like $p 21, G A D D 45, c-m y c$ etc

\section{PIV-02 BRCA2 interacting proteins}

H Vidarsson, E Mikaelsdottir, JE Eyfjörd, H Ögmundsdóttir, T Rafnar and S Valgeirsdóttir

Laboratory for Molecular and Cell Biology, Icelandic Cancer Society, Reykjavik, Iceland

Two breast cancer susceptibility genes (BRCA1 and $B R C A 2)$ have been cloned. A BRCA2 founder mutation, BRCA2 999del5, accounts for the majority of familial breast cancer cases in Iceland. Individuals heterozygous for this mutation are at an increased risk of developing breast cancer, but the risk varies between families, indicating variable penetrance. BRCA2 encodes a protein of 3418 amino acids with a molecular weight of $384 \mathrm{kDa}$. The biological function of BRCA2 is, as yet, not well known, but several studies have shown that BRCA2 interacts with proteins like
RAD51 and P/CAF, known to be involved in DNA damage response pathways and DNA transcription in cells. In an effort to elucidate the cellular function of BRCA2, we have studied the interaction between BRCA2 and other cellular proteins in mammary epithelial cells. The focus of our study has been on interaction with other transcriptional factors in the nucleus. Preliminary data indicate that BRCA2 interacts with Stat proteins upon ligand stimulation. Identification of proteins that interact with BRCA2 can shed light on its physiological role in the development of breast cancer. 
PIV-03 Embryonic lethality in the Brca1-1700T mouse model suggests inhibition of p53-dependent pathways

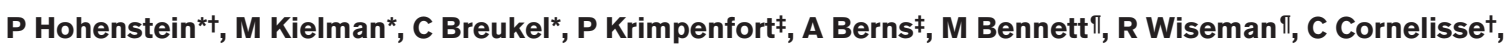
G-J van Ommen*, P Devilee*+ and R Fodde*

${ }^{*}$ MGC Department of Human Clinical Genetics, LUMC, Leiden, ${ }^{\dagger} P G D$ Department of Pathology, LUMC, Leiden, ${ }^{\ddagger}$ Department of Molecular Genetics, Netherlands Cancer Institute, Amsterdam, The Netherlands, ^Laboratory of Molecular Carcinogenesis, NIH, Research Triangle Park, North Carolina, USA

The vast majority of mutations found in the human BRCA1 gene predict expression of a truncated protein, which may retain one or more functional domains. To test the possibility that truncated BRCA1 proteins might have a dominant effect on wild-type BRCA1 or other proteins, we generated a targeted mouse model, designated Brca11700T, in which a neomycin resistance gene is inserted in exon 20 of the murine Brca1 gene. This mutation is predicted to result in expression of a mutant Brca1 protein which will not encompass the last BRCT repeat, most likely resulting in disruption of the p53-specific transactivation domain. RT-PCR analysis confirmed that the mutant transcript is expressed.

Mice heterozygous for this mutation do not show a predisposition to tumorigenesis. Also $\gamma$-radiation does not have a significant effect on these mice. As has been reported for the other Brca1 mouse models, homozygosity for this mutation is embryonic lethal. However, the developmental stage at which lethality occurs differs from the other models. Preliminary results indicate that homozygous Brca11700T embryos can reach headfold stage, although not before $9.5 \mathrm{dpc}$, after which further development is blocked or lethally impaired.

The observed embryonic phenotype resembles the one found in compound Brca1-/-Tp53-/- embryos. This might suggest that in the Brca11700T mouse model embryonic lethality is caused by a combination of disturbed dsDNA break repair and an impaired p53-dependent response to the resulting genetic instability. Possibly, p53 binds to the mutant Brca1 protein, which still contains the p53-binding domain. However, since the C-terminal p53 transactivation domain is likely to be disturbed, this interaction might result in p53 being blocked in an inactive complex. Recently, p53 was shown to be able to lead to tumorigenesis by haploinsufficiency. The latter observation suggests that even a partial sequestering of $\mathrm{p} 53$ by a mutant Brca1 protein may result in a cellular phenotype.

PIV-04 Heterogeneity in the clinical phenotype of TP53 mutations in breast cancer patients

J Alsner*, M Yilmaz*, P Guldberg ${ }^{\dagger}$, LL Hansen* and J Overgaard*

${ }^{*}$ Aarhus University Hospital, Aarhus, ${ }^{\dagger}$ Danish Cancer Society, Copenhagen, Denmark

TP53 mutation is a strong independent marker for survival in breast cancer with some heterogeneity in the clinical phenotype of various types of mutations. Based on 315 patients with breast carcinoma, we suggest a new model for the differentiation of TP53 mutations. Although TP53 mutation in general was associated with aggressive tumour/patient characteristics, missense mutations outside any conserved or structural domain did not affect the clinical outcome (risk of disseminated disease and death). In contrast, patients with missense mutations affecting amino acids directly involved in DNAor zinc-binding displayed a very aggressive clinical phenotype. Null mutations (including missense mutations disrupting the tetramerization domain) and the remaining missense mutations displayed an intermediate aggressive clinical phenotype. When patients were divided into 3 groups, (i) wild-type together with the missense mutations outside structural/conserved domains, (ii) null mutations and the missense with intermediate clinical phenotype, and (iii) the very aggressive missense mutations, disease-specific survival rates were $89 \%, 58 \%$, and 35\% (5-year actuarial values, $P<0.0001$ ), respectively. In a Cox proportional hazards analysis, separation of TP53 mutations according to these criteria eliminated the prognostic importance of all investigated classical factors except nodal status.

\section{PIV-05 The GSTT1 genotype is associated with p53 mutation status of breast cancer tumours}

\section{K Gudmundsdóttir, L Tryggvadóttir and JE Eyfjörd \\ The Icelandic Cancer Society, Reykjavík, Iceland}

The role of the glutathione S-transferases is to provide protection against reactive mutagenic electrophiles by catalysing their conjugation to glutathione. In humans there are four classes of cytosolic GSTs, $\alpha$ (GSTA), $\mu$ (GSTM), $\pi$ (GSTP) and $\theta$ (GSTT). Homozygous deletions of $50 \%$ and $20 \%$ of the genes coding for GSTM1 and GSTT1, respectively, results in conjugation deficiency. An A-G polymorphism at nucleotide 313 results in an amino acid substitution (lle105Val) in the substrate binding site of the GSTP1 gene. The risk to the individual carrying a 
variant of one of these genes is estimated to be low, but the high frequency in the population of some of these variants makes the population attributable risk high. The aim of this study is to investigate whether any associations exist between the above mentioned GST genotypes and breast cancer, and whether they affect the p53 mutation status of the tumours and penetrance of germline mutations in the BRCA2 gene. The polymorphisms have been analysed by PCR, electrophoresis and RFLP. No significant differences have been observed between the GST genotypes in the 258 controls and 450 cases analysed so far. Both groups will be enlarged to increase the power of the study. p53 mutations in breast cancer tumours are significantly increased in individuals carrying the GSTT1 null genotype, both alone and in combination with the GSTP1 AG/GG genotype. The effect of the GST genotypes on the penetrance of $B R C A 2$ has to be studied further.

\section{PIV-06 Studies of TP53 haplotypes in relation to LOH and TP53 mutations in breast cancer patients}

A Langerød*, TI Andersen*†, I Bukholm*‡ and A-L Børresen Dale*

${ }^{*}$ Department of Genetics, Institute for Cancer Research, The Norwegian Radium Hospital, ${ }^{+}$Present address: Department of Oncology, Ullevaal University Hospital, Oslo, Norway, ${ }^{\ddagger}$ Present address: Department of Surgery, Akershus Central Hospital, Lørenskog, Norway

Case-control studies have reported association between polymorphisms in the TP53 gene and breast cancer [1]. We have examined whether certain alleles or haplotypes show association with loss of heterozygosity (LOH) or mutations in TP53. Our hypothesis is that certain alleles may predispose for breast cancer through a mechanism promoting $\mathrm{LOH}$ or mutations. 452 breast cancer patients were genotyped for three intergenic polymorphisms [2-4] and one polymorphism located downstream of the gene [5]. The SNPs (Single Nucleotide Polymorphisms) in exon 4 and intron 6 were analysed using the restriction enzymes BstUl and Mspl respectively, while the $16 \mathrm{bp}$ insertion in intron 3 and the VNTR downstream of the gene were examined using capillary electrophoresis. $\mathrm{LOH}$ and mutation analyses have previously been performed in samples from the same cohort. In conclusion, we were not able to demonstrate any statistical significance implying that any of these polymorphisms were associated with increased risk of $\mathrm{LOH}$ or mutation of the TP53 gene.

\section{References}

1. Weston A, Pan CF, Ksieski HB, et al: Cancer Epidemiol Biomarkers Prev 1997, 6:105-112.

2. Ara S, Lee PS, Hansen MF, Saya H: Nucleic Acids Res 1990, 18:4961.

3. Lazar V, Hazard F, Bertin F, Janin N, Bellet D, Bressac B. Oncogene 1993, 8:1703-1705.

4. McDaniel T, Carbone D, Takahashi T, et al: Nucleic Acids Res 1991, 19:4796.

5. Jones MH, Nakamura Y: Genes Chromosomes Cancer 1992, 5:89-90.

\section{PIV-07 P53 mutations in breast and ovarian carcinomas from BRCA1 and 2 mutation carriers and noncarriers}

\section{E Sensi, C Ghimenti, G Cipollini, D landolo, G Naccarato, P Viacava, G Bevilacqua and MA Caligo}

Department of Oncology, Division of Pathology, University of Pisa, Italy

Breast and ovarian carcinomas occurring in carriers of $B R C A 1$ and 2 gene mutations may have a distinct pathway of molecular pathogenesis from those occurring in noncarriers. Data from murine models suggest that the p53 gene, which is involved in initiating cell cycle arrest and apoptosis in response to DNA damage, may be important in the tumorigenesis of BRCA1 and 2-associated cancers, and its loss of function could be a early critical event in the malignant transformation of cells defective for $B R C A 1$ and 2 genes. Therefore, breast and ovarian tumors from carriers of $B R C A 1$ and 2 alterations might be expected to exhibit a high rate of somatic p53 mutations.

An analysis was carried out on 84 Italian hereditary breast and/or ovarian families to evaluate the frequency of $B R C A 1$ and 2 mutations by PTT and PCR-SSCP. 21 out of 84 families showed disease-associated $B R C A$ germline mutations: 15 probands (18\%) had BRCA1 mutations and 6 patients $(7 \%)$ presented alterations in the BRCA2 gene. In addition, $80 \%$ of mutations found in the $B R C A 1$ gene and $33 \%$ of alterations in the $B R C A 2$ result in a premature termination of translation. The frequency of p53 mutations was then evaluated in 40 tumor DNAs from 33 out of 84 families analysed for $B R C A 1$ and 2 gene alterations (8 were $B R C A 1$ mutation-carriers and 25 were noncarriers).

The tumor DNAs were screened for alterations in the DNA-binding domain of the p53 gene (exon 5 through 8) using PCR-SSCP. Direct sequencing was performed on gene fragments that showed altered mobility in the PCRSSCP pattern. Mutations in the $p 53$ gene were detected in 4 out of 8 tumors from $B R C A 1$ and 2 mutation carriers $(50 \%)$ versus 2 out of 32 carcinomas from noncarriers (6\%). All the observed mutations were single-base-pair substitutions. One alteration affected the splice donor site of exon 6 , and it was present in a BRCA-negative family as germline mutation. The expression of p53 protein in $B R C A$-carrier and noncarrier tumors was analysed by immunohistochemistry using the mouse monoclonal antibody DO-7. The same analysis was also performed in a consecutive series of 72 sporadic tumors as a control group. 5 out of $8 B R C A$-carrier tumors (63\%) and 2 out of 
Breast Cancer Research Vol 2 Suppl 1 The Second International Symposium on the Molecular Biology of Breast Cancer

32 BRCA-noncarrier carcinomas $(6 \%)$ were positive for p53 staining. Finally, 25 out of 72 sporadic tumors (35\%) had p53-positive immunostaining. A significantly higher frequency of p53 mutation and overexpression was found in the $B R C A$-associated tumors. Our data are in keeping with the postulate that loss of p53 checkpoint control is important in the molecular pathogenesis of breast and ovarian carcinomas in carriers of BRCA1 and 2 mutations.

\section{PIV-08 Screening breast cancer patients for Norwegian ATM mutations}

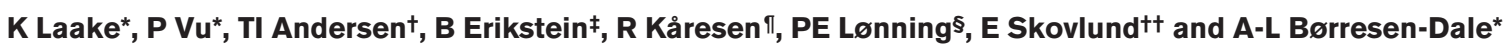

${ }^{*}$ Department of Genetics, The Norwegian Radium Hospital, Oslo, ${ }^{+}$Department of Oncology, Ullevål University Hospital, Oslo,

${ }^{\ddagger}$ Department of Oncology, The Norwegian Radium Hospital, Oslo, "Department of Surgery, Ullevål University Hospital, Oslo,

$\S$ Department of Oncology, Haukeland Hospital, Bergen, ${ }^{++}$Clinical Research Office, The Norwegian Radium Hospital, Oslo, Norway

Heterozygosity for Ataxia Telangiectasia (AT), a cancerprone recessive syndrome, has been associated with an increased risk of breast cancer. In the present study, 483 Norwegian breast cancer patients were screened for carrier status of six different ATM mutations found in Norwegian AT patients. One breast cancer patient carried the Norwegian founder mutation, giving a point estimate of the frequency of $0.2 \% \quad(95 \% \quad \mathrm{Cl}$ $0.01-1.2 \%)$. Assuming a $0.5 \%$ carrier frequency, the present results are consistent with a maximum 2.4-fold increased lifetime risk of breast cancer in ATM heterozygotes. The study had 95\% power to detect a 4.6 -fold elevated lifetime risk, and a 9-fold elevated risk in women below age 55 .

PIV-09 Constitutional alterations of the ATM gene in early-onset sporadic breast cancer

P Maillet*, H Bonnefoi ${ }^{*}$ and A-P Sappino*

*Identification of Genetic Predispositions to Cancer Unit, Division of Oncology, ${ }^{+}$Gynecology and Obstetric Department, HCUG, 1211 Geneva 14, Switzerland

Since epidemiological evidence suggests that obligate ataxia telangiectasia (A-T) heterozygotes are at increased risk of developing breast cancer, we have analysed the germline configuration of the ataxia telangiectasia mutated gene (ATM) in 26 premenopausal breast cancer patients with no familial history of breast/ovarian cancer and who developed breast cancer before the age of 40 . Five previously undescribed germline sequence variants were detected by SSCP screening of the 66 ATM exons. These included 3 rare variants with an estimated allelic frequency of less than

1\%: IVS59-20del4, IVS63+24delTT, and K1454N; 1 rare polymorphism (IVS56+23insT) with an estimated allelic frequency of $2 \%$; and 1 missense mutation F1463C. We considered F1463C as a pathogenic mutation because the same phenylalanine amino acid substituted for a serine at this position is a known A-T mutation. No sequence variant was found in a control group of 45 healthy blood donors. These observations support the hypothesis that constitutional alterations of the ATM gene may contribute to the pathogenesis of some early-onset sporadic breast cancer.

PIV-10 Modulation of ATM gene expression in a murine mammary epithelial cell line

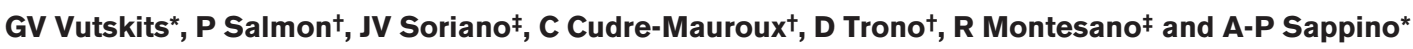

${ }^{*}$ Division of Oncology, Department of Internal Medicine, HUG, 24, rue Micheli-du-Crest, 1211 Geneva 14, ${ }^{+}$Department of Genetics and Microbiology and ${ }^{\ddagger}$ Department of Morphology, University of Geneva Medical School, CMU, 1, rue Michel-Servet, 1211 Geneva 4, Switzerland

To explore the role of atm in epithelial cell biology, we have used an in vitro model, in which non-transformed TAC-2 murine mammary epithelial cells recapitulate features of normal breast epithelium differentiation, when grown in a three-dimensional collagen gel. To modulate atm gene expression or impair atm function we designed antisense and dominant negative constructs which were delivered into TAC-2 cells through conventional plasmids. However, since these vectors failed to achieve effective long-term transgene expression, we have then used lentiviral vectors to express our constructs in TAC-2 cells with greater efficiency. We plan to use this model to assess the impact of atm dysfunction on the following parameters: cell proliferation and survival, cell cycle progression and morphogenetic properties. These investigations are expected to better delineate the contribution of the atm gene as a cancer-predisposing gene in the breast and to provide further insights into our understanding of atm function in epithelial cell biology. 


\section{PIV-11 Loss of heterozygosity on chromosome arm 16q in breast cancer: clinical, molecular and} statistical approaches

A-M Cleton-Jansen*†, HM van Beerendonk*, NT ter Haar*, PHC Eilers ${ }^{\ddagger}$, HC van Houwelingen ${ }^{\star}$, BA Bonsing $^{\star}$, VTHBM Smit*, G-JB van Ommen ${ }^{\dagger}$ and CJ Cornelisse*

Departments of ${ }^{*}$ Pathology, ${ }^{\dagger}$ Human and Clinical Genetics and ${ }^{\ddagger}$ Medical Statistics, Leiden University Medical Center, Leiden, The Netherlands

Loss of heterozygosity ( $\mathrm{LOH})$ on the long arm of chromosome 16 is one of the most frequent genetic events in breast cancer, occurring in at least $50 \%$ of the breast cancer cases. It is one of the earliest genetic events, occurring already in the pre-invasive stage, in ductal carcinoma in situ. Our study on flow-sorted tumour populations shows that chromosome $16 q$ loss occurs in the DNA diploid subpopulation of tumour cells, before aneuploidization, which is also indicative of a role in early tumorigenesis.

A detailed $\mathrm{LOH}$ analysis using 30 highly polymorphic markers and careful revision of the histology of a series of 200 primary invasive breast tumours could not demonstrate any significant correlation between $\mathrm{LOH}$ on $16 \mathrm{q}$ and histopathological markers. On the contrary there was a markedly higher number of cases with $16 \mathrm{q} \mathrm{LOH}$ in a series of well differentiated pre-invasive breast cancers when compared with poorly differentiated pre-invasive tumours. This suggests a difference in time of occurrence of $16 \mathrm{q} \mathrm{LOH}$ in these two morphological distinguishable groups, ie early LOH in well differentiated tumours and late (upon invasion) in the poorly differentiated subset.

Since mapping of the smallest region of deletion involved in $\mathrm{LOH}$ has not yet resulted in identification of the tumour suppressor genes which are the target of $16 \mathrm{q} \mathrm{LOH}$, we have applied a novel statistical approach for the analysis of our data using an improved version of the model described previously by Newton et al (Statist Med 1998, 17:1425). The program has been improved by introducing the possibility for the existence of more than one tumour suppressor locus and by increasing its speed by some orders of magnitude. Analysis of $16 \mathrm{q} \mathrm{LOH}$ data in breast cancer with this program provides evidence for the presence of two different tumour suppressor loci on chromosome arm 16q.

\section{PIV-12 Analysis of Fhit expression in stages of breast cancer progression}

M Campiglio*, G Somenzi*, P Aiello*, F Wärnberg ${ }^{\dagger}$, L Holmberg $^{\dagger}, \mathrm{CM}^{\mathrm{C}} \mathrm{Croce}^{\ddagger}$ and S Menard*

*Molecular Targeting Unit, Department of Experimental Oncology, Istituto Nazionale Tumori, Milan, Italy, ${ }^{\dagger}$ Department of Surgery, University Hospital, Uppsala, Sweden, ${ }^{\ddagger}$ Kimmel Cancer Institute, Thomas Jefferson University, Philadelphia, PA, USA

The tumour suppressor gene FHIT, encompassing the FRA3B fragile site on chromosome $3 p 14.2$, is more than $1 \mathrm{Mb}$ in size and encodes for a $1.1 \mathrm{~kb}$ cDNA. It belongs to the histidine triad (HIT) superfamily and encodes a cytoplasmic $16.8 \mathrm{kDa}$ protein. Epithelial cells in most human tissues strongly express Fhit protein, while Fhit expression is absent or reduced in a large fraction of tumours. Fhit protein reduction or absence occurs in $70 \%$ of breast cancer specimens, suggesting that alteration of Fhit expression in this tumour is a frequent event, caused by both alterations in the regulation of Fhit expression and by the well documented biallelic deletion of the gene. To determine how Fhit down-regulation influences breast cancer progression, we have examined protein expression at different stages of the disease. Starting from normal epithelia, we have also considered morphological lesions of various grades, such as atypical ductal hyperplasia (ADH), in situ breast carcinoma (DCIS) and neoplasia. Preliminary data indicated that a decrease or absence in Fhit protein expression is associated with high proliferation and large tumour size. Electron microscopy analysis has revealed that Fhit protein is organised into small cytoplasmic clumps, mainly confined to the end of a polymerised tubulin and to the plasma membrane extroversion, suggesting a possible role of Fhit in cytoskeleton structures.

Supported by AIRC.

PIV-13 Analysis of chromosome alterations and the E-cadherin and FHIT genes in lobular breast cancer

\section{S Ingvarsson, C Huiping, JG Jonasson and BA Agnarsson}

Department of Pathology, University Hospital, Reykjavik, Iceland

We have studied a set of 40 human lobular breast cancer for $\mathrm{LOH}$ at various chromosome locations, including intragenic FHIT markers at chromosome 3p14.2, and for mutations of the E-cadherin gene. A significantly lower level of $\mathrm{LOH}$ was detected at chromosome arms, 1p, 3p, 9p, 11q, $13 q$ and $18 q$ in lobular compared to ductal breast tumours.
On the contrary, all lobular cases were found with $\mathrm{LOH}$ at chromosome 16q22.1, containing the E-cadherin locus. A significant association was detected between $\mathrm{LOH}$ at $3 p$ and high $S$ phase, $\mathrm{LOH}$ at $9 p$ and low ER and PgR content, and between $\mathrm{LOH}$ at $17 \mathrm{p}$ and aneuploidy. $\mathrm{LOH}$ within the FHIT gene was detected in $16 \%$ of the lobular 
cases, which is significantly lower than detected in ductal breast cancer. A significant association was found between LOH at the FHIT gene and reduced Fhit expression detected by IHC. The expression of Fhit was reduced to a similar level in lobular and ductal breast cancer. Thus, genetic alterations within the FHIT gene leading to loss of Fhit proteins may play an important role in the carcinogenesis of a significant number of lobular breast cancers, even though the frequency of alterations is lower than in ductal breast cancer. Six novel mutations were detected within the E-cadherin gene in combination with $\mathrm{LOH}$ of the wild type E-cadherin locus and reduced E-cadherin expression. $\mathrm{LOH}$ at $13 q$ was significantly associated with $\mathrm{LOH}$ at $7 \mathrm{q}$, $\mathrm{LOH}$ at $11 \mathrm{q}$ and $\mathrm{E}$-cadherin gene mutations. We conclude that there is a molecular difference in the pathogenesis of lobular and ductal breast cancer.

\section{PIV-14 Excluding TTC4 as a target for loss on chromosome 1p31 in human breast cancer}

\section{Y Hey, W Brintnell, L James and J Varley}

Cancer Genetics Group, Paterson Institute for Cancer Research, Wilmslow Road, Manchester, UK

We have previously reported a region of high loss of heterozygosity in human breast cancer on chromosome 1p31.1. Recently a new member of the human tetratricopeptide repeat-containing gene family, TTC4, was mapped to a YAC 879a6 which encompasses the smallest region of overlapping loss (SRO) reported by our group. This therefore became a candidate for a new breast cancer tumour suppressor gene. We used multiple pairs of PCR primers from the gene to screen CEPH and Zeneca YACs covering the region, but were unable to amplify a product from any of them, including two independent isolates of YAC 879a6. We have isolated both a BAC (31C23) and YAC (13EA7) using primers from the $3^{\prime}$ untranslated region of TTC4. In single and double FISH experiments both 13EA7 and 31C23 located on chromosome 1p but distal to $879 \mathrm{a} 6$ at $1 \mathrm{p} 31.3$. This localisation was confirmed by screening a panel of monochromosome hybrids. Comparison of TTC4 sequence with the genome database identified a match between the $3^{\prime}$ untranslated region of the gene and EST WI-9676. However, this EST was assigned to chromosome 7 by radiation hybrid mapping, transcript and YAC contig mapping. We therefore identified YACs from these contigs using primers from Wl-9676 and sequenced the resulting PCR products. These revealed a number of nucleotide alterations that suggested that the sequence on chromosome 7 is a pseudogene. Finally pseudogene-specific primers were used to identify two new BACs, one of which $(51 \mathrm{H} 8)$ was localised to $7 \mathrm{p} 13-14$ by FISH. In conclusion, we have therefore reassigned TTC4 by FISH to $1 \mathrm{p} 31.3$, excluding it as a target for inactivation in human breast cancer at $1 \mathrm{p} 31.1$, and identified a TTC4 pseudogene that maps to chromosome 7p13-14.

PIV-15 A detailed physical map of a region within human chromosome 16q22.1

\section{E Frengen*, P Rocca-Serra†, S Shaposhnikov*, L Taine ${ }^{\ddagger}$, J Thorsen*, C Bépoldin†, M Longy†, H Prydz*} and $\mathrm{F}$ Dorion-Bonnet ${ }^{\dagger}$

*The Biotechnology Centre of Oslo, University of Oslo, Norway, ${ }^{+}$Laboratoire d'Oncologie Moléculaire, Institut Bergonié, Bordeaux, France, ${ }^{\ddagger}$ Laboratoire de Cytogénétique, Centre Hospitalier Universitaire Pellegrin, Bordeaux, France

We have previously described a tight cluster of five apparently unrelated genes on human chromosome 16q22.1. An expanded region surrounding this gene cluster has now been mapped using P1 artificial chromosome clones (PACs). This PAC map is currently used to identify and characterize new genes from the q22.1 region of human chromosome 16. Work is also underway to reveal the functions of selected genes in the contig.

The construction of the contig was performed by using probes derived from the end of the starting PACs in repeated library screening. If the region mapped contains large duplicated sequence elements, this chromosome walking could potentially lead to the extension of the map into unlinked chromosomal regions. Such large duplicated sequences of several tens of kilo base pairs, which are shared by several human chromosomes, have previously been reported. To verify the organization of the PAC map, we have therefore performed long-range mapping experiments. High molecular weight human genomic DNA was digested with a panel of rare-cutting restriction enzymes, separated by PFGE, blotted and hybridised with selected probes from the contig. These results demonstrated that the contig faithfully represents the chromosomal region covered by the PACs. Furthermore, clusters of restriction sites for CpG cutters are strong evidence for the presence of CpG islands, which are landmarks for genes. Therefore, the mapping experiments have also resulted in the identification of several genes within human chromosome $16 \mathrm{q} 22.1$.

This work is supported by grants from The Norwegian Research Council and The Norwegian Cancer Society (EF and HP), and The Comite du Lotet-Garonne de la Ligue Nationale Contre le Cancer (ML and FDB). PRS was a recipient of an ARC fellowship. 


\section{PIV-16 Expression of variant forms of the MUC1 gene correlates with the invasiveness of breast cancer} cells

R Zeillinger, B Schmid, D Tong, B Fasching, I Schiebl, M Stimpfl, A Obermair and S Leodolter

University of Vienna, Department of Obstetrics and Gynecology, Division of Gynecology, Molecular Oncology Group, A-1090

Vienna, Waehringer Guertel 18-20, Austria

The characterization of tumor markers is of prime importance in understanding the mechanisms underlying cancer initiation and progression. The most exclusively used marker for monitoring breast cancer patients are the protein products of the MUC1 gene, which is strongly overexpressed in breast cancer cells. The best characterized MUC1 gene product is MUC1/REP (episialin, PEM, CA15-3, MCA). It is important in reducing cell-cell and cell-extracellular matrix interactions, probably being involved in the spread of cancer cells from the primary tumor. MUC1 overexpression was found to correlate with invasiveness. Four isoforms are generated by differential splicing due to the use of alternative splice acceptor sites for exon 1. These were designated variants $A$ to $D$. $A$ higher expression of variant $A$ than of variant $B$ was found to indicate thyroid papillary carcinomas.

We investigated the expression of these variant forms in 23 permanent breast cell lines. RNA samples were ana- lyzed by RT-PCR and subsequent automated quantitative fragment analysis. The cell lines were also analyzed for invasiveness by an in vitro collagen invasion assay. Ten cell lines showed invasive growth, either as single cells or as cell clusters. Variant A was solely expressed in four of the invasive cell lines and was preferentially expressed in one line (ratio 35.1), whereas only 1 out of 13 non-invasive cell lines expressed more variant $A$ than variant $B$ (ratio 1.1). This correlation between the mRNA expression of variant $A$ and the in vitro invasiveness was statistically significant $(P=0.035)$. Moreover, variant $D$ was concomitantly found with the preferentially expressed variant $A$.

This is the first report about the correlation of expression of a MUC1 splice variant and the invasiveness of breast cancer cells. We conclude that not only overexpression of MUC1 in cancer cells is responsible for metastasis, but also the expression of variant forms.

PV-01 p16INK4A as a predictive factor in patients with locally advanced breast cancer treated with neoadjuvant doxorubicin monotherapy

S Geisler*, B Smith-Sørensen†, D Betticher ${ }^{\ddagger}$ IK Bukholm+币, LA Akslen§s, A Kappeler ${ }^{\ddagger}$, M Gugger ${ }^{\dagger+}$, PE Lønning* and A-L Børresen-Dale ${ }^{\dagger}$

${ }^{*}$ Department of Medicine, Section of Oncology and § Pathology, Haukeland University Hospital, Bergen, ${ }^{\dagger}$ Department of Genetics, The Norwegian Radiumhospital, Oslo, Norway, ${ }^{\ddagger}$ Institute of Medical Oncology and ${ }^{+\dagger}$ Pathology, University of Bern, Switzerland

The cyclin-dependent kinase inhibitor p16 (p16 INK4A/ CDKN2A) binds to Cdk4 and inhibits the formation of the Cdk4/cyclin D1 complex, thereby inhibiting the cyclin D-dependent phosphorylation of the retinoblastoma protein $(p R b)$. Phosphorylation of $p R b$ leads to release of the $\mathrm{E} 2 \mathrm{~F}$ transcription factor that activates genes promoting cell cycle progression from $\mathrm{G} 1$ to $\mathrm{S}$-phase. Alterations of $p 16^{\text {INK } 4 A}$, leading to its inactivation, result in the deregulation of cell proliferation through loss of $\mathrm{G} 1$ arrest control, and can thereby contribute to the formation of cancer and may influence tumour response to chemotherapy. To investigate the role of $\mathrm{p} 16^{\mathrm{INK} 4 \mathrm{~A}}$ as a predictive factor in the neoadjuvant treatment of patients with breast cancer, we have analysed the $\mathrm{p} 16$ status in a series of 91 patients treated for locally advanced breast cancer with doxorubicin monotherapy. We measured p16 INK4A protein expression with use of immunohistochemistry, studied possible mutations by direct sequencing of exon 1 and 2, and determined the methylation status of $\mathrm{CpG}$ sites in exon $1 \alpha$. Of 90 tumours examined by immunostaining, 28 were negative or expressed p $16^{\mathrm{INK} 4 \mathrm{~A}}$ at low levels (grade $1=<5 \%$ positively stained tumour cells), 35 had a moderate p16 INK4A expression (grade $2=5-50 \%$ positively stained tumour cells), and 27 had strong expression of $16^{\text {INK } 4 \text { A }}$ (grade $3=>50 \%$ positively stained tumour cells). One tumour had a missense mutation in codon 145 in addition to methylation of exon $1 \alpha$ (p16 INK4A immunostaining grade 3 ), and three tumours displayed methylation of exon $1 \alpha$ ( 2 showed p16 $16^{\text {INK } 4 \mathrm{~A}}$ immunostaining grade 1 , and 1 showed $p 16^{I N K 4 A}$ immunostaining grade 2). One tumour with methylation of exon $1 \alpha$ has previously been reported to have a mutation of TP53 affecting the L2/L3 domains. p16/NK4A methylation correlated with lack of response to doxorubicin treatment; $2 / 4$ patients with $\mathrm{p} 16^{\mathrm{INK} 4 \mathrm{~A}}$ methylation progressed on therapy, compared to 7/86 without p16 INK4A methylation $(P=0.048)$. On the contrary, p16 INK4A immunostaining did not correlate with treatment response, nor with immunostaining for $\mathrm{pRb}, \mathrm{p} 1 \mathrm{gARF}$, cyclin D1 and cyclin E, nor mutational analyses for TP53. Our data suggest that $\mathrm{p} 16^{\mathrm{INK} 4 \mathrm{~A}}$ alterations may be involved in chemoresistance in breast cancer, although immunostaining alone fails to show a predictive value for response to doxorubicin treatment. 


\section{PV-02 Low frequency of $p 16^{I N K 4 a}$ promoter methylation in mammary carcinomas as revealed by positive} display of methylated sites

R Lilischkis, $\mathrm{H}$ Kneitz and H Kreipe

Institute of Pathology, Hannover Medical School, Hannover, Germany

Promoter methylation represents an important mechanism for silencing gene expression in higher eukaryotes. In order to study methylation of the promoter of the tumour suppressor $p 16^{I N K 4 a}$, we developed a fast and simple method that in contrast to previous studies relies on the positive display of methylated sites (PDM). The method is based on bisulphite treatment of DNA, PCR-amplification of the modified DNA, and restriction digest of de novo created restriction sites to positively display DNA methylation in a background of unmethylated DNA. Since methy- lated as well as unmethylated DNA is amplified, information on the proportion of both is provided.

Using this approach, we analysed 33 ductal invasive mammary carcinomas, 4 normal mammary tissues and 4 cell lines for methylation. p16 INK $4 \mathrm{a}^{\mathrm{m}}$ methylation was detected in $1 / 33$ carcinomas (3\%) and in 0/4 normal tissue samples. We conclude that PDM provides a useful tool for determining the degree and pattern of promoter methylation and is suitable for screening large series of tissue samples.

\section{PV-03 Estrogen-dependent C-jun upregulation may control cyclin D1 expression}

\section{Planas-Silva, J Liu Donaher and RA Weinberg}

Whitehead Institute for Biomedical Research, Cambridge, Massachusetts, USA

Estrogen controls the proliferation of estrogen-receptor positive breast cancer cells. In an effort to understand how estrogen promotes cell-cycle progression we and others have found that expression of the cell-cycle regulator cyclin D1 is tightly controlled by estrogen in MCF-7 cells. However, stable expression of the estrogen receptor in different cell lines is not sufficient to allow estrogen-dependent cyclin D1 expression. This lack of cyclin D1 upregulation in cells stably expressing estrogen receptor (ER) may explain why estrogen cannot induce proliferation in these cells. To further understand the molecular mechanisms by which cyclin D1 is regulated in response to estrogen, we have characterised in more detail the response of $\mathrm{HaCaT}$ cells expressing ER to estrogen, and compared them with those observed by MCF-7. Differential activation of AP-1 members is seen after estrogen treatment of MCF-7. This MCF-7 specific upregulation of c-fos and c-jun precedes and correlates well with cyclin D1 induction by estrogen. Further studies using the cyclin D1 promoter indicate that cjun upregulation by estrogen may induce cyclin D1 expression and most likely cell cycle progression. Therefore, we suggest that the ability of MCF-7 cells to activate c-jun in response to estrogen is crucial to understanding the estrogen-dependent proliferation of breast cancer cells.

PV-04 Negative regulation of p53 expression is an essential, rate-limiting function of Jun in the control of cell cycle progression

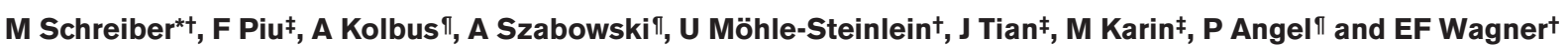

*University of Vienna, Department of Obstetrics and Gynecology, Division of Senology, Vienna, Austria, ${ }^{\dagger}$ Research Institute of Molecular Pathology (IMP), Vienna, Austria, ${ }^{\ddagger}$ University of California at San Diego, Department of Pharmacology, La Jolla, CA, USA, "Deutsches Krebsforschungszentrum (DKFZ), Heidelberg, Germany

The tumor suppressor gene p53 is inactivated by mutations in $>50 \%$ of human tumors, including breast cancers. Here we show that p53 expression is negatively regulated by the Jun proto-oncogene, which encodes a component of the mitogen-inducible immediate-early transcription factor AP-1 and has been implicated as a positive regulator of cell proliferation. In fibroblasts derived from Jun-/- mouse fetuses, the tumor suppressor gene p53 and its target gene, the CDK inhibitor p21, are expressed at elevated levels, whereas overexpression of Jun represses p53 and p21 expression. Surprisingly, protein stabilisation, the common mechanism of p53 regulation, does not seem to be involved in upregulation of p53 in Jun-/- fibroblasts. Rather, Jun was found to negatively regulate transcription of $\mathrm{p} 53$ by direct binding to a conserved AP-1 site in the p53 promoter. Furthermore, overexpression of Jun accelerates cell proliferation, whereas the absence of Jun results in a severe proliferation defect and a prolonged crisis prior to spontaneous immortalisation. The cyclin D1- and cyclin E-dependent kinases (CDKs) and transcription factor E2F are poorly activated, resulting in inefficient G1 to S-phase progression. Importantly, deletion of p53 abrogates all defects of Jun-/- cells in cell cycle progression, proliferation, immortalisation, and activation of G1 CDKs and E2F. These results demonstrate that the sole rate-limiting function of Jun in fibroblast proliferation is negative regulation of p53 expression, and establish a mechanistic link between Jun-dependent mitogenic signalling and cell cycle regulation. 


\section{PV-05 Setting up an efficient and reliable screening method for novel c-Jun $\mathbf{N}$-terminal kinase} substrates

T Kallunki*, C Holmberg*, M Lerdrup-Hansen*, T Herdegen†, A Aronheim and M Jäättelä*

${ }^{*}$ Danish Cancer Society, Copenhagen, Denmark; ${ }^{+}$Christian-Albrechts University of Kiel, Germany; ${ }^{* B}$ Rappaport Faculty of Medicine, Technion-Israel Institute of Technology, Israel

The c-Jun N-terminal kinase (JNK), also known as the stress activated protein kinase (SAPK), forms a family of serine-threonine kinases that can be efficiently activated by both mitogenic and apoptotic signals. Moreover in various cases JNK activation has been shown to have both preventative and causative roles in apoptosis. Thus far the best characterized target of JNK is c-Jun, which forms a part of the transcription factor AP-1. It is a well established fact that the activation of JNKs in the cell will lead to the phosphorylation of Ser63 and Ser73 at the c-Jun activation domain. This in turn results in the transcriptional activation of the AP-1 responsive genes. We show here that, in some cancer cell lines, JNK activation does not always correlate with AP-1 activation. This lack of AP-1 activation is also associated with the lack of the phosphorylation of c-Jun. We have been testing two different 'substrate screening systems' in order to find novel, relevant JNK substrates from these cancer cells.

\section{PVI-01 Angiogenesis in breast cancer: establishing a link between angiogenesis and hormone} regulation

R Soares, C Costa, F Gärtner and F Schmitt

Institute of Molecular Pathology and Immunology of the University of Porto - IPATIMUP, Porto, Portugal

Angiogenesis is a process of formation of new blood vessels that is essential for tumour growth and metastasis. There is recent evidence indicating that angiogenesis can be regulated by hormones. The aim of our study was to evaluate the effect of oestrogen in angiogenesis using a hormone-dependent cancer model, breast cancer. We studied two different breast cancer cell lines (one hormonedependent, MCF7, and one hormone-independent, Hs578T), that were inoculated in the mammary fat pad of nude mice. The mice were treated with oestrogen and the tumours were removed when they reached $80 \mathrm{~mm}^{3}$. Angiogenic index, VEGF and TGF $\alpha$ were evaluated by immuno- histochemistry and Western blotting. The MCF7 tumours had a higher microvessel density and expressed both VEGF and TGF $\alpha$. In contrast, Hs578T, xenografted in mice, presented a lower angiogenic index, expressed VEGF, but did not express TGF $\alpha$. We also studied a series of 86 human breast carcinomas and demonstrated a significant association between TGF $\alpha$ and angiogenic index: TGF $\alpha+$ cases, $48.1 \pm 28.0 ; \quad$ TGF $\alpha-$ cases, $31.8 \pm 20.0$ microvessel density $(P=0.01)$. Since by binding to its receptor, oestrogen induces the transcription of TGF $\alpha$, our results suggest that TGF $\alpha$ is a putative factor linking hormone regulation and angiogenesis in breast cancer.

\section{PVI-02 Development of a high throughput, molecular diagnostic assay for predicting telomerase activity} in breast cancer cell lines and tissues

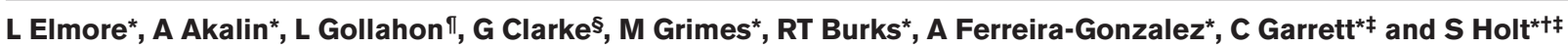

${ }^{*}$ Departments of Pathology and ${ }^{+}$Human Genetics, ${ }^{\ddagger}$ Massey Cancer Center, Medical College of Virginia Campus at Virginia

Commonwealth University, Richmond, VA, ^Department of Biological Sciences, Texas Tech University, Lubbock, TX; §Baylor

College of Medicine, Houston, TX, USA

Telomerase is a cellular enzyme that helps to provide genomic stability in tumor cells by maintaining the integrity of telomeres. Telomerase is an RNA-dependent DNA polymerase that contains a protein component (hTERT) and an associated RNA (hTR), which is used as a template for telomere repeat addition. Telomerase activity, while not detectable in most normal human somatic cells, is associated with approximately $85 \%$ of malignant human cancers overall, including over $90 \%$ of breast cancers.

We have optimized a novel, quantitative, high-throughput telomerase activity assay using fluorescently labelled primers and Real Time quantitation via the $A B I$ Prism 7700 (a.k.a., the TaqMan). Using established breast cancer cell lines and a subset of breast tumors, we demonstrate that telomerase levels quantitated from the TaqMan-based assay closely correlate with values obtained using the traditional, gel-based telomerase activity assay (TRAP). In addition, we have assessed the levels of both hTERT mRNA and hTR in each of our samples via RT-PCR to determine whether relative amounts or a ratio of the two telomerase components correlate with activity in a given sample. Our ultimate goal is to develop a Real Time, fluorescent RT-PCR assay to simultaneously measure hTERT and hTR messages in breast tumor samples, in an attempt to convert the enzymatic telomerase activity assay into a quantitative nucleic acid test to predict levels of activity in routinely processed clinical specimens. 


\section{PVI-03 Mammary epithelial cells immortalized by over-expression of the catalytic subunit of human telomerase may represent a model for mammary epithelial differentiation}

J DiRenzo, R Rivera-Gonzalez and M Brown

Dana Farber Cancer Institute, Boston MA, USA

Retroviral transfer of a cDNA encoding human Telomeric End Reverse Transcriptase (hTERT) into primary human mammary epithelia has led to the establishment of several clonally derived lines of Immortalized Mammary Epithelial Cells (IMECs). Unlike their empty vector control counterparts, the IMECs were capable of bypassing replicative senescence. In doing so, they exhibited a marked decrease in the protein levels of the retinoblastoma gene product, $\mathrm{Rb}$, and a complete loss of the cyclin-dependent kinase inhibitor p16, events which are hallmarks of the immortalization process. In culture, IMECs proliferate in a manner that is dependent on insulin and Epidermal Growth Factor (EGF). Interestingly, these IMECs can be induced to undergo a differentiation which is characterized by an arrest of the cell cycle in G1 and the loss of cyclin D1 expression. During this differentiation process, IMECs establish cell-cell interactions that lead to an ordered arrangement of cells in two dimensions. Further genetic and biochemical characterization may hopefully reveal the nature of these differentiated IMECs.

PVII-01 Molecular analyses of human breast cancer metastasis: genetic markers of progression

\section{K Driouch and $\mathbf{R}$ Lidereau}

\section{Centre Rene Huguenin, St. Cloud, France}

Breast tumorigenesis and metastasis result from an accumulation of genetic alterations involving 'cancer genes'. The prognostic value of these genetic alterations has been greatly investigated. However, few of them have been studied in secondary tumors, owing to the limited availability of surgical specimens. In human cancers, the genetic mechanisms underlying the metastatic process are still poorly understood. We investigated whether certain recurrent alterations could be associated with the metastatic process. We analysed the genetic profiles of primary tumors, local recurrences, and distant metastases of breast cancer. The alteration profiles in these 3 types of samples were different, suggesting distinct mechanisms of progression.

$M Y C, E R B B 2$ and $C C N D 1$ amplification is rare in distant metastases of breast cancer. These genetic amplifications could be involved in the genesis of primary tumors, but less in the later stages of breast cancer progression. In contrast, $\mathrm{LOH}$ is a frequent genetic event in breast cancer metastases. The $\mathrm{LOH}$ regions frequently observed in primary breast tumors are also detected in breast cancer metastases, mostly due to a clonal evolution of metastatic cells from the primary site to the metastases, but specific altered regions could also be acquired during metastatic progression. $\mathrm{LOH}$ analyses have defined regions of deletion associated with metastasis on several chromosomal regions, ie 3p21.3, 7q31, 15q14, 16q22.1 and 16q23.2. These regions contain several candidate metastasis suppressor genes such as DLC1, RPL14, DEF3, CTNNB1, MET, THBS1, CDH1. Other metastasis-related genes such as NME1 and KAl1 show losses of expression that do not correlate with $\mathrm{LOH}$. Other genetic mechanisms could be involved. These studies could lead to the characterisation of new genomic markers of tumor aggressiveness and enhance our understanding of the molecular mechanisms of metastasis and cancer progression.

\section{PVII-02 AKT-1 and BCL-2 co-expression in breast cancer patients correlates with better survival}

\section{G Pérez-Tenorio, O Stål and the Southeast Sweden Breast Cancer Group}

Department of Oncology, Faculty of Health Sciences, Linköping University, Linköping, Sweden

Background and purpose: Akt-1 is a serine/threonineprotein kinase that regulates growth factor-dependent cell proliferation and survival. Activated-Akt-1 causes $\mathrm{Bcl}-2$ release from the BAD:Bcl-2 inactive complex. Bcl2 is not only able to prevent apoptosis, as a downstream effector of Akt-1, but also can delay cell-cycle progression. Akt-1 is over-expressed in breast cancer cell lines and tumours, while $\mathrm{Bcl}-2$ has been related with tumour survival and drug resistance in vitro and to an $\mathrm{ER}+/$ well differentiated sub-group of tumours, in vivo. Since endocrine treatment effectiveness could be due to activation of the apoptotic program, we wanted to investigate the expression and relationship between these factors as well as other variables (C-erbB-2, ER, and S-phase).

Patients and methods: Frozen tissue from primary tumours of 104 breast cancer patients (age $<50$ years), who received tamoxifen, zoladex or both (follow-up period $>10$ years), was used to determine the expression of Bcl-2 and Akt- 1 by immunohistochemistry. C-erbB-2 expression and S-phase were analysed using flow cytometry. The statistical analysis was performed using the 'Statistica' package.

Results: There was a positive correlation between $\mathrm{Bcl}-2$ and Akt-1 expression (38 Akt-1+/65 Bcl-2+ compared 
with 11 Akt-1+/37 Bcl-2- cases, $P=0.007)$. This correlation also appears in metastasis-free patients $(P=0.0008)$ but not in those patients with metastasis $(P=1.0)$. Bcl-2 alone was not significantly associated with ER, S-phase or C-erbB-2 expression $(P>0.05)$ but a trend was observed for Bcl-2-positive cases to present ER+/low-S-phase/CerbB-2-negative phenotypes. In terms of distant-recurrence-free survival, those patients expressing Akt-1+/
$\mathrm{Bcl}-2+$ survived longer than Akt-1+/Bcl-2- patients $(P=0.028)$ while no benefit was observed for Bcl-2 $(P=0.6)$ or Akt-1 $(P=0.9)$ individually.

Conclusions: The results suggest that Akt-1 might reinforce the prognostic value of $\mathrm{Bcl}-2$, and probably this phenotype characterises a subgroup of patients less prone to undergo metastasis.

\section{PVII-03 Does a polymorphism in the CYP17 gene predict mammographic density?}

\section{G Ursin, SA Ingles, H Spencer Feigelson, GA Coetzee, L Bernstein, MC Pike and A Buley}

University of Southern California, Preventive Medicine, Norris Comprehensive Cancer Center, 1441 Eastlake Avenue, Los Angeles, CA 90089, USA

Mammographic density has been associated consistently with breast cancer risk in epidemiological studies. We and others have shown that hormonal status is associated with mammographic density. A pilot study was conducted to determine if a known polymorphism in a gene, CYP17, involved in the biosynthesis of female sex steroids, plays a role in mammographic density. The CYP17 gene encodes the cytochrome P450c17 enzyme which mediates the $17 \alpha$-hydroxylation of pregnenolone and progesterone, and subsequent conversion of these 17-hydroxylated products to the estradiol precursors DHEA and androstenedione. In the 5'-UTR region of the CYP17 gene, a C/T single nucleotide polymorphism (SNP) results in two alleles designated $\mathrm{A} 1$ and $\mathrm{A} 2$. The $\mathrm{A} 2$ allele has been associated with higher circulating hormone levels in both premenopausal and postmenopausal women.

We used data from 94 breast cancer cases aged 40 to 64, diagnosed between 1994 and 1998, who partici- pated in the Los Angeles part of the population-based Women's Contraceptive and Reproductive Experience (CARE) case-control study. We obtained blood samples from the cases, and scanned mammograms obtained prior to diagnosis (some as long as 5 years before). Mammographic density was determined using a method we have published previously. Subjects were genotyped for the CYP17 SNP using genomic DNA from blood.

The mean percent density in the three groups of women with the $A 1 A 1, A 1 A 2$ and $A 2 A 2$ alleles were similar (25.4\%, 25.3\% and 29.3\%). However, compared with women with the A1A1 genotype, the odds ratios (and $95 \%$ confidence intervals) of having a dense mammogram $(>50 \%$ mammographic density) in women with the $\mathrm{A} 1 \mathrm{~A} 2$ or A2A2 genotype were $3.9(0.8-19.3)$ and $5.6(0.8-9.6)$ respectively, $(P$ for trend $=0.09$ ). The results suggest that CYP17 may be associated with mammographic density, possibly via its effect on estrogen levels.

\section{PVII-04 Ribozymes targeting the tumor suppressor BRCA1 lead to increased chemosensitivity to cisplatin and etoposide and chemoresistance to microtubule-interfering agents in HBL100 breast cancer cell line}

\section{S Lafarge, M Ferrara and YJ Bignon \\ Laboratory of Molecular Oncology EA2145, Centre J Perrin, BP392, 58 rue Montalembert, 63011 Clermont-Ferrand Cedex1, France}

$B R C A 1$ is a cancer susceptibility gene involved in about $45 \%$ of the familial breast cancers and $80 \%$ of families predisposed to breast and ovarian cancer. BRCA1 codes for a protein of 1863 amino acids with multiple functions, including DNA repair, activation of transcription, control of cellular proliferation and involvement in apoptosis. In keeping with these diverse functions, $B R C A 1$ interacts with several proteins including hRad51, complex hRad50-hMre11-p95/nibrin, and gamma-tubulin. We developed an antisense strategy using hammerhead ribozymes against this gene to understand the roles and the functions of BRCA1. As BRCA1 is involved in DNA repair, our first goal is to evaluate the sensitivity of ribozyme-treated cells to chemotherapeutic drugs.

We designed and tested 3 ribozymes in cell-free extracts. We cloned the two most efficient ribozymes into the retrovi- ral vector LXSN and transfected them by lipofection in the HBL100 cell line. We performed Real-Time quantitative PCR to measure the expression of BRCA1 mRNA vs $18 \mathrm{~S}$ rRNA, and examined the protein level by western blot analysis. We have found four clones with an inhibition of BRCA1 mRNA expression between 82 and 99\% which correlated with the protein level. We tested the sensitivity of these clones to different drugs (doxorubicin, etoposide, cisplatin, taxol and vincristine) by the sulforhodamine $B$ proliferation test. The decrease in expression of BRCA1 led to sensitivity to DNA damaging agents (cisplatin and etoposide) and resistance to microtubule-interfering agents (MIA) (taxol and vincristine). No difference was found for doxorubicin treatment.

Our results with etoposide and cisplatin are consistent with the role of $B R C A 1$ in DNA repair. The results with 
MIA are more unexpected. The observed chemoresistance may be related to the interaction of BRCA1 with gamma-tubulin, a component of the mitotic spindle. The function of BRCA1 in this pathway must be investigated further to understand this modification of chemosensitivity.

\title{
PVII-05 Cytokeratin and mammaglobin as tumor markers in patients with high risk breast cancer
}

\author{
PF Ferrucci, C Rabascio, C Corsini, F Tealdo, M Paolucci, F Bertolini, H El Taani and G Martinelli \\ European Institute of Oncology, Milan, Italy
}

Aims of the study: To investigate the incidence and prognostic relevance of tumor micro-contamination in BM and/or G-CSF-mobilized peripheral blood progenitor cells collections of stage II-III breast cancer patients.

Patients and methods: Patients were enrolled from September 1998 through May 1999 and underwent high-dose chemotherapy with autologous PBPC transplantation. We analysed a total of 71 patients for TC on PBPC apheresis (28 of them had previously frozen samples). 43/71 patients also had their BM evaluated on the day of the first PBPC collection after 5 days of G-CSF administration (BM-STIM) and immediately before HDCT (BM-PRECT). Cytokeratin (CK) expression was evaluated in all samples by either immunocytochemistry (ICC, sensitivity $1 \times 10^{-6}$ cells, $9-16 \times 10^{-6}$ cells for each sample) and reverse transcriptase nested PCR (RT-PCR, sensitivity $1 \times 10^{-7}$ cells). Amplified products were then annealed to a ${ }^{32} \mathrm{P}$-labelled internal sequence probe to confirm specificity. 41 patients were also evaluated by nested RT-PCR for Mammaglobin (MAM, sensitivity $1 \times 10^{-6}$ cells) gene expression.

Results: (1) PBPC frequency of CK+ was $11 \%$ by ICC and $66 \%$ by RT-PCR on 71 patients' samples; (2) BMSTIM and BMPRECT frequency of CK+ was 7-14\% by ICC and $65-60 \%$ by RT-PCR on 43 patients' samples; (3) all CK ICC+ samples were MAM RT-PCR+; (4) 53\% of patients with CK RT-PCR+ BM-STIM had their PBPC and BM-PRECT CK RT-PCR+; (5) after a median followup of 21 months on 28 patients with frozen samples, $25 \%$ of patients relapsed and $43 \%$ of them had contaminated PBPC; (6) 74\% of CK ICC+ samples were CK RT-PCR+; (7) BMSTIM, PBPC and BMPRECT frequencies of MAM+ were 15,17 and $22 \%$ respectively by RT-PCR on 41 patients' unfrozen samples; (8) PBPC frequency of MAM+ was $20 \%$ by RT-PCR on 25 patients' frozen samples.

Conclusion: (1) MAM gene evaluation could add a lot of sensitivity and specificity to the overall results. (2) G-CSF administration for PBPC mobilization does not increase PBPC contamination. (3) A longer follow-up of these patients is needed to evaluate the prognostic relevance of different markers of tumor cell contamination in HDCT for breast cancer.

\section{PVIII-01 Promising method for DNA extraction from paraffin embedded archive material}

\section{A Bregård, P Vu, G Geitvik and A-L Børresen-Dale \\ Department of Genetics, The Norwegian Radium Hospital, Oslo, Norway}

Formalin-fixed, paraffin-embedded materials are an invaluable source for mutation analysis. However, DNA extraction from this type of material can be challenging as well as time consuming. DNA extracted from paraffin-embedded material is often highly fragmented and contaminated by protein agents. For DNA analysis, such as PCR (polymerase chain reaction), subsequent TTGE (temporal temperature gradient electrophoresis) and DNA sequencing, optimal conditions require long DNA fragments (200-1000 bp) and a DNA with high purity with an OD ratio (abs. at $260 \mathrm{~nm} / \mathrm{abs}$ at 280 $\mathrm{nm}$ ) between 1.6 and 2.0. We have evaluated and combined different protocols to get the highest quality and yield of DNA. $5-10 \mathrm{~mm} \times 8-10$ sections of tissue were used.

The best results were achieved from extractions using relatively high volumes $(8-10 \mathrm{ml})$ of xylene and ethanol for the deparaffinization and rehydration steps initiating the extraction protocol. Furthermore, limiting the incubation period for proteinase $\mathrm{K}$ digestion of the material to 4-8 hours yields longer fragments of DNA than prolonged digestion. This, however, demands a prolonged incubation period with lysis buffer, up to 24 hours, previous to digestion. The phenol/chloroform extraction step in the traditional extraction procedure contains several uncertain elements, risking protein contamination from the interphase between the aqueous and organic phases, and the phenol health hazards are also considerable. Using a PLG (phase lock gel) tube from Eppendorf, in which a gel plug separates the organic phase and the aqueous phase, greatly eases the extraction and increases DNA yield and purity. The organic phase is locked underneath the gel, leaving no room for protein contamination when pipetting off or decanting the upper, aqueous phase. The health risk posed by the solvent vapour released during the isolation of the aqueous phase is also minimised by the gel barrier. Subsequent salt precipitation with $1 \mathrm{M} \mathrm{NaCl}$ and ethanol 'rinse' is performed before the samples are air-dried and diluted in 100-200 $\mu \mathrm{l} 1 \times$ TE buffer. DNA yield and quality were evaluated by a spectrophotometer, a fluorometer and PCR fragments separated on an agarose gel followed by EtBr staining. The OD ratio $260 / 280 \mathrm{~nm}$ of the extracted DNA was 1.67-1.97 for different batches. Six out of 10 
samples yielded PCR products with fragments as long as 770 bp. A multiplex PCR for 6 exons of the ATM gene was performed with success. Previously extracted DNA from the same type of tissue block using different protocols yielded no PCR products for the same multiplex PCR.
This reliable method of extraction, although a bit time consuming, makes analysis of paraffin-embedded material possible, yielding satisfactory results for further study of the DNA. This protocol will now be used for detection of ATM mutation carriers among family members of AT children who have died of cancer.

\title{
PVIII-02 REAL Real-Time!
}

\section{F Boeckman, L Tan and K Hamby}

Bio-Rad Laboratories, 2000 Alfred Nobel Drive, Hercules, CA 94547, USA

Recent advances in real-time PCR analysis have led to significant improvements in the accuracy and simplicity of DNA quantification and gene expression analysis. Here we demonstrate the new Bio-Rad iCycler iQ system capabilities. When PCR is conducted on 96 replicates, we achieve a uniformity with a CV of less than $1 \%$, consistent with that of other well-known systems for real-time PCR analysis. We demonstrate the ability to distinguish a two-fold dilution series of human genomic DNA down to 125 genomic equiva- lents. We also show a wide dynamic range over which quantification is possible, beginning with plasmids or genomic DNA. The iCycler iQ system is designed to work with many detection strategies; here we show the iCycler iQs' ability to employ a variety of techniques, including SYBR Green I, TaqMan ${ }^{\circledR}$ and Molecular Beacons. Finally, the iCycler iQ's unique ability to analyse data at any point within a cycle or dwell time can be a significant advantage when evaluating certain detection chemistries including molecular beacons.

\section{PVIII-03 Exact quantification of gene amplification in archival tissue sections by laser-assisted microdissection and real-time PCR}

\author{
U Lehmann, S Glöckner, W Kleeberger, R von Wasielewski and H Kreipe \\ Institute of Pathology, Medizinische Hochschule Hannover, D-30625 Hannover, Germany
}

Gene amplification is one of the most important mechanisms leading to deregulated gene expression in cancer. The exact quantitative detection of this frequent genomic alteration in solid tumors is hampered by admixture of non-neoplastic bystander cells. In order to overcome this shortcoming and to develop an objective quantification method, we have combined laser-based microdissection of tumor cells with the novel $5^{\prime}$-exonuclease based real-time PCR-assay that enables the highly reproducible exact quantification of minute amounts of nucleic acids. As a model system, amplification of the c-erb-B2/Her-2/neu gene and the adjacent topoisomerase $/ / \alpha$ gene were determined in paraffin-embedded breast cancer tissue $(n=23)$ after immunohistochemical labelling and laser-based microdissection.

The quantitative assay was linear over a broad range approaching the theoretical detection limit. 91\% (21/23) of the specimens were suitable for the PCR analysis. The immunohistochemical labelling of cells did not interfere at all with the quantitative PCR. The high sensitivity of realtime PCR enabled the reliable and objective detection of low level amplifications in as few as $\mathbf{5 0}$ cells from archival tissue sections. In selected cases intratumor heterogeneity was analysed using areas of approx. 50-100 cells. In addition, we have already started the systematic analysis of gene amplification in DCIS of the breast to correlate morphological classification systems with the results of molecular analysis.

This novel approach, combining immunohistochemistry, laser-microdissection and quantitative kinetic PCR, allows morphology-guided studies in archival tissue specimens and will enable the exact quantification of gene copy numbers even in small and precancerous lesions.

\section{PVIII-04 Screening for BRCA1 gene mutations by capillary electrophoretic REF-SSCP}

\section{P Kringen*, HG Eiken ${ }^{\dagger}$, JC Pedersen*, S Egedal*, TE Ottesen*, K Berg*, KM Tveit ${ }^{\ddagger}$ and TI Andersen*}

Departments of ${ }^{*}$ Medical Genetics and ${ }^{+}$Oncology, Ullevål University Hospital, N-0407 Oslo, ${ }^{\ddagger}$ Center for Molecular Medicine, Haukeland Hospital, N-5031 Bergen, Norway

The identification of novel mutations in large genes requires efficient mutation-scanning techniques. Except for DNA-sequencing and PTT (protein truncation test), SSCP (single strand conformation polymorphism) is the technique that has been the most extensively applied to scan for mutations in the BRCA1-gene. In the present study a capillary electrophoretic (CE) restriction endonuclease fingerprinting (REF) modification of the SSCP technique was established for BRCA1 exon 11.

Samples containing a total of 16 known nucleotide changes in BRCA1 exon 11 were examined. Exon 11 was 
amplified in four overlapping PCR-fragments. Aliquots of labelled PCR products were submitted to digestion with three or four fragment-specific restriction enzymes and submitted to electrophoresis. Each sample was analysed using (1) radioactive labelling and polyacrylamide gel electrophoresis (PAGE) and (2) fluorochrome labelling and capillary electrophoresis in an $\mathrm{ABI} 310$ sequencer. Samples giving abnormal electropherograms were reamplified and sequenced to identify the exact nature of the nucleotide change. All of the 16 known nucleotide changes could be detected by method 1 . The aberrant band indicating the presence of one of the mutations (G484X) was, however, difficult to reproduce. All nucleotide changes but $\mathrm{G} 484 \mathrm{X}$ were detected by fluorochrome-labelled CE-REF-SSCP (method 2). This method appeared as the faster and technically more convenient of the two.

CE-REF-SSCP was chosen as the mutation-scanning technique in our further $B R C A 1$ studies. A series consisting of 75 affected members of Norwegian breast cancer families was first screened for a set of Norwegian BRCA1 mutations using restriction enzyme-based tests. The samples were then screened for novel mutations in exon 11 by CE-REF-SSCP. The results of this mutation screening will be presented.

\section{PVIII-05 Hypervariable area in the $5^{\prime}$ flanking region of GSTP1, previously reported as a minisatellite repeat}

V Nedelcheva Kristensen*, T Kristensen† and A-L Børresen-Dale*

${ }^{*}$ Department of Genetics, Institute of Cancer Research, The Norwegian Radium Hospital, Montebello 0310, Oslo, ${ }^{+}$Department of Biochemistry, University of Oslo, Norway

Glutathione $S$ transferases are dimeric molecules, catalysing the conjugation of activated (acetylated, alkylated, hydroxylated, oxygenated) molecules of xenobiotics to glutathione. Large deletions in the genes coding for some of the enzymes (GSTM1, GSTT1) are known and related to deficiency in conjugation of the metabolites of xenobiotics. Alterations in glutathione metabolism have been shown to have an important impact on the cytotoxicity of various free radical-producing anticancer drugs, like adriamycin. The overexpression of GSTP1 was shown to be involved in the acquisition of resistance to anticancer drugs like adriamycin, cisplatin, melphalan and etoposide.
Two polymorphisms in GSTP1 are known: a point mutation in exon 5 with a possible functional role, leading to changes in the kinetic properties of the enzyme, and a repeat of AAAAT in the $5^{\prime}$ untranslated region immediately upstream of an extensively methylated $\mathrm{CpG}$ island. Poly AT-rich repeats are implicated as differential enhancers of transcriptional activation. Here we report that the repeat in the AT-rich area of the $5^{\prime}$ untranslated region is further degenerated by insertions of CAC, ATT and other motifs, and describe a detailed analysis of the polymorphism with a putative role in the regulation of transcriptional activation.

\section{PVIII-06 Comparative genomic hybridisation analysis of myoepithelial carcinoma of the breast}

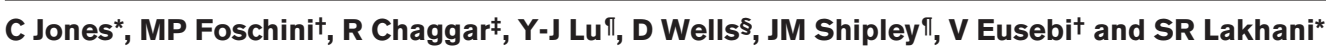

${ }^{*}$ Departments of Histopathology, ${ }^{S}$ Obstetrics and Gynaecology, and ${ }^{\ddagger}$ The Ludwig Institute for Cancer Research, University College London, UK, 'Sezione di Anatomia, Istologia e Citologia Patologica 'M. Malpighi', Università di Bologna, Italy, "Section of Cell and Experimental Pathology, The Institute for Cancer Research, Surrey, UK

Although there appears to be a common stem cell for the two epithelial cell types in the breast, the majority of breast cancers exhibit a luminal phenotype. Pure myoepithelial carcinomas are rare. We report our findings of genetic alterations in these tumours. We have analysed 10 cases of pure spindle cell myoepithelial carcinomas using laser capture microdissection and comparative genomic hybridisation. The mean number of changes was 2.1 (range 0-4), compared to a mean of 8.6 (range 3.6-13.8) in unselected ductal carcinomas. Common alterations included loss at $16 q$ ( $3 / 10$ cases), $17 p$ (3/10), 11q (2/10) and 16p (2/10), regions also com- monly deleted in ductal carcinomas. The single case in which both pure myoepithelial carcinoma and invasive ductal carcinoma was present showed two alterations in the myoepithelial tumour (losses at $17 p$ and $17 q$ ), while the invasive ductal component showed fourteen alterations (5 gains and 9 losses), including loss at $17 p$. The sharing of $17 p$ loss in myoepithelial and ductal carcinoma is consistent with a common stem cell model in the breast. The relatively few genetic alterations in otherwise aggressive neoplasms suggests that myoepithelial tumours may be a good model for the delineation of genes important in breast tumorigenesis. 


\title{
PVIII-07 Genetic alterations in ductal carcinoma in situ and invasive carcinoma of the breast
}

\section{E Robanus-Maandag, C Bosch, C Vos, H Peterse and $\mathbf{M}$ van de Vijver}

The Netherlands Cancer Institute, Amsterdam, The Netherlands

Several histological classifications propose a subdivision of ductal carcinoma in situ (DCIS) into well-, intermediately, and poorly differentiated subtypes. The use of biological parameters (extent of immunopositivity, proliferation rate, and aneuploidy) facilitates such subdivision. Moreover, determination of genetic alterations can contribute to the identification of the different DCIS subtypes. Our recent data indicate that inactivation of an unidentified tumor suppressor gene on chromosome $16 \mathrm{q}$ is involved in the development of most well and intermediately differentiated DCIS. Moreover, amplification and inactivation of various genes on chromosome 17 are implicated in the development of poorly differentiated DCIS. These data show that there is a genetic basis for the classification of DCIS in a well and poorly differentiated type, and support the evidence of independent genetic routes to develop a specific type of carcinoma in situ of the breast.

Our study has revealed that the spectrum of genetic alterations in the in situ tumors is comparable to that of the invasive carcinomas. However, the frequencies of the individual genetic alterations differ significantly between the two tumor categories. As most invasive carcinomas also contain an in situ component, we want to compare the genetic alterations in both components of the same tumor and, in this way, identify the genetic alterations that are involved in the progression from the in situ to the invasive stage. We have microdissected the invasive and adjacent in situ component of paraffin-embedded invasive breast carcinomas with a relatively large in situ component, isolated DNA, and performed comparative genomic hybridization (CGH).

\section{PVIII-08 Identification of two novel breast cancer associated genes by the differential display method}

\author{
N Maass ${ }^{\star \dagger}$, K Nagasaki $^{\dagger}$, VR Jacobs* and W Jonat* \\ ${ }^{*} \mathrm{OB} / \mathrm{GYN}$, Department of Oncology, University of Kiel, Germany, ${ }^{\dagger}$ National Cancer Center, Tokyo, Japan
}

Using the Differential Display (DD) technique we identified two novel genes that are over-expressed in human breast cancer cells compared to various other human cancer cells which were screened for differentially expressed genes.

Messenger RNAs were transcribed, followed by PCR amplification and visualisation of the cDNA subpopulation by polyacrylamide gel electrophoresis. Eight human tumor cell lines where used to select differentially expressed genes by DD. Cloning and sequencing of two overexpressed cDNA clones in MCF 7 breast cancer cells identified two novel genes. By $\mathrm{FISH}$ analysis one gene was mapped on the $X$ chromosome and therefore designated 'breast cancer associated gene on chromosome X' (BG-
X). The second novel gene, designated DAM1 (DNA amplified in mammary carcinoma) was mapped on the chromosome 1p13.3-21 region, which is frequently altered in human breast cancer. Northern blot analysis of BG-X revealed ubiquitous expression in normal human tissue (eg kidney, pancreas, small intestine, stomach, colon, ovary, liver, brain, heart) and in breast cancer cells (7/7), but no expression in several human cancer cells (carcinomas of pancreas (1/9), stomach $(0 / 7)$ and prostate $(0 / 4))$. Using an enhanced green fluorescent protein (EGFP) assay, the EGFP-BG-X-fusion protein was localised in the cell nucleus. Our data present two novel genes with strong expression in human breast cancer cells and down-regulation in several other cancer cell lines.

\section{PVIII-09 Expression profiling of BRCA1 associated breast tumors}

\section{EMJJ Berns, IL van Staveren, L Verhoog, JA Foekens and JGM Klijn}

Department of Medical Oncology, Division of Endocrine Oncology, JNI Be424, PO Box 1738, 3000DR Rotterdam The Netherlands

BRCA1-associated breast tumours frequently show unfavourable features, ie poor differentiation, high proliferation indices, aneuploidy, ER- and PgR negativity, and TP53 positivity. These data are based on single gene analysis. Expression arrays, however, allow for the simultaneous investigation of multiple genes. We have used Atlas Human Cancer cDNA Expression Arrays (Clontech), on which 588 cancer-related genes are spotted, for an exploratory analysis. Profiles of one cell line and six tumours from patients with an inherited BRCA1 gene mutation were weighed against those from 15 patients without a family history who had similar clinico-pathological characteristics which are col- lected in our computerised database system. Total RNA isolation was performed according to standard procedures. RNAs were used to synthesise ${ }^{32} \mathrm{P}$-radiolabeled cDNA for hybridisation to the cancer cDNA expression arrays, according to the manufacturer's instructions. Data were acquired and quantified using the Molecular Dynamics Phospholmager and ImageQuant software (Molecular Dynamics, Sunnyvale, USA). The levels of the lowest and highest expressed genes differed at 100- or 1000-fold. In an exploratory analysis we have considered only the upper 30\% ranking of the signals for each tumor sample as 'high' expression $(H)$, and the data were dichotomised: high $(H)$ vs low $(L)$. 
In this pilot study on $6 B R C A 1$ and 15 sporadic tumours we observed that 14 genes showed high $(\mathrm{H})$ expression levels, in all cases. Furthermore, 396 genes showed a heterogeneous expression pattern (including the EGF-R, MYC, p16, HER2/neu and UPA). These heterogeneous expression levels are consistent with our previous studies on breast cancer. Ten genes are mostly expressed at an increased level in BRCA1 tumours when compared to sporadic tumours. Interestingly the majority of these genes are known to play a role in cell adhesion, motility and invasion. Although the series of breast tumours analysed is relatively small, we were able to identify genes whose expression appears differential in BRCA1 and sporadic breast tumours. Cluster analysis, which allows for grouping of tumors and genes according to similar patterns of expression, revealed that the expression profiles of 5 out of $6 B R C A 1$-associated tumors are clustered in one arm. Extension of the number of genes and tumour samples will reveal additional genes.

\section{PVIII-10 Transcript profiles and genotyping of cancer tissue}

\section{Malmqvist*, M Sievertzon*, A Gustafsson ${ }^{*}$, A Holmberg*, M Larsson*, A Alderborn', M Uhlén ${ }^{*}$ and J Lundeberg* \\ *Department of Biotechnology, KTH, Royal Institute of Technology, Stockholm, ${ }^{+}$Pyrosequencing AB, Uppsala, Sweden}

Gene expression profiling has become one of the most attractive approaches to elucidate gene function. For these purposes, hybridization methods and SAGE have been the most commonly used techniques. We have developed an alternative strategy for cDNA tag analysis that results in a quantitative estimate of gene expression. The strategy relies on generation of $3^{\prime}$-tagged cDNA libraries and a new non-gel-based high throughput DNA-sequencing principle, pyrosequencing. Pyrosequencing is based on a sequencing by synthesis strategy in which single specific nucleotides are added to an extension substrate in the presence of a DNA polymerase. Incorporation is detected in real-time through an enzymatic cascade that produces a quantitative light signal, measured by a CCD-camera. A microtiter format is used, allowing sequencing of 96 samples within $40 \mathrm{~min}$. In total, 2000 clones from a human tissue model system have been analysed by both conventional DNA sequencing and pyrosequencing. For the analysis of only a few cells, a cDNA amplification step, keeping the relative transcript levels, is used in the generation of the libraries. Furthermore, an SNP analysis technique based on pyrosequencing and the p53 tumor suppressor gene has been developed, that will allow correlation between expression and genotype of cancer tissue. The quantitative gene expression profiles from compared libraries are visualized by virtual chip technology.

\section{PVIII-11 Relation of gene expression-based tumor subclasses to clinical phenotypes}

\section{T Sørlie, CM Perou, PE Lønning, PO Brown, D Botstein and A-L Børresen-Dale}

The Norwegian Radium Hospital, Oslo, Norway

Gene expression analysis by cDNA microarrays is a powerful tool for characterizing the variation in transcriptional programs in cells and tissues. We have analysed surgical specimens from 40 human breast tumors using cDNA microarrays representing 8000 human genes. From 20 of the tumors, pairs of biopsies were obtained both before and after a 16-week course of doxorubicin chemotherapy. Two of the tumors were paired with lymph node metastases. Expression patterns reflecting specific features of physiological variation and cellular composition of the tumors provided unique molecular portraits of each tumor. Different clusters of co- expressed genes, like the estrogen receptor, the ERBB2 gene and basal cell specific genes, were used to further subclassify the tumors. The different subgroups, identified by unique expression patterns, were associated with clinical parameters such as overall survival. Also, the TP53 mutation status in the tumors was associated with disease outcome within these subclasses. Although the sample size in any of the subgroups is too small to support any statistically robust tests, our preliminary results show the potential of gene expression-based subtyping of breast cancer in predicting clinical behaviour and therapy response. 